\title{
A systematic review and meta-analysis of optical coherence tomography in multiple sclerosis
}

\author{
Axel Petzold MD * Johannes F. de Boer $\mathrm{PhD}^{\dagger}$ \\ Sven Schippling MD $\$$ Patrik Vermersch MD $\$$ \\ Randy Kardon MD ^ Ari Green MD\| \\ Peter A. Calabresi MD ** Chris Polman MD *
}

July 7,2010

*VU Medical Center, Department of Neurology, Amsterdam, The Netherlands
$\dagger$ Institute LaserLaB Amsterdam Department of Physics, VU University, De Boelelaan
1081, 1081 HV Amsterdam, The Netherlands
$\ddagger$ Institute for Neuroimmunology and clinical Multiple Sclerosis Research (inims), Uni-
versity Medical Center Hamburg Eppendorf, Martinistrae 52, 20246 Hamburg, Germany
\$University of Lille Nord de France, department of Neurology, Lille, France
ॠlowa City VA Center for Prevention and Treatment of Visual Loss, Department of
Ophthalmology and Visual Sciences Department of Veterans Affairs Hospital lowa City,
lowa USA 52242
"Multiple Sclerosis Center, Department of Neurology, University of California San
Francisco, San Francisco, CA 94143 , USA
** Johns Hopkins Hospital, $600 \mathrm{~N}$ Wolfe St, Pathology 627, Baltimore, MD 212187 , USA 


\begin{abstract}
Optical coherence tomography (OCT) is a new method potentially applicable for the analysis of neurodegeneration in multiple sclerosis (MS) by capturing thinning of the retinal nerve fibre layer (RNFL). Metaanalyses of time domain OCT (TDOCT) data demonstrates RNFL thinning of $20 \mu \mathrm{m}\left(95 \% \mathrm{Cl} 18-23, \mathrm{n}=2063, \mathrm{p}_{i} 0.00001\right)$ following $\mathrm{MS}$ optic neuritis (MSON) and $\mu \mathrm{m}\left(95 \% \mathrm{Cl} 6-9, \mathrm{n}=3154, \mathrm{p}_{i} 0.00001\right)$ in MS without MSON. The estimated RNFL thinning in patients with MS is above what is expected from normal ageing. The likely cause being retrograde trans-synaptic degeneration and progressive loss of retinal ganglion cells. The RNFL correlated with visual and neurological functioning as well as with paraclinical data. Promising developments in order to better understand the structurefunction relationship in MS pathophysiology include spectral/Fourierdomain OCT (SD/FDOCT) technology, polarisation sensitive OCT, fluorescence labelling, structural assessment of action potential propagation and segmentation algorithms allowing for quantitative assessment of different retinal layers.
\end{abstract}

Word count: abstract 145, main text 5340.

Keywords optical coherence tomography, retinal nerve fibre layer, axonal loss, neurodegeneration, multiple sclerosis 


\section{Contents}

1 Introduction 4

2 Methods 5

3 OCT in Multiple Sclerosis $\quad 6$

4 Time course of RNFL loss in MS 10

5 OCT and disability in MS 12

5.1 Visual function . . . . . . . . . . . . . . . . . . 13

5.2 Global clinical scores . . . . . . . . . . . . . . . . . . . 16

6 OCT and electrophysiology in MS 18

6.1 VEP . . . . . . . . . . . . . . . . . . 18

6.2 ERG . . . . . . . . . . . . . . . . . 19

$\begin{array}{lll}7 & \text { OCT and imaging in MS } & 20\end{array}$

8 Future developments $\quad 20$

9 Conclusion $\quad 24$ 


\section{Introduction}

Optical coherence tomography (OCT) is a non-invasive technique ${ }^{1}$ which allows for an "optical biopsy" of accessible tissues such as the retina (Figure 1). Over the past decade OCT matured into an interesting, highly sensitive tool for imaging of neurodegeneration in multiple sclerosis (MS) research. ${ }^{2,3}$ Because the retina is the only place where a tissue layer made up of axons can be imaged directly, quantification of the retinal nerve fibre layer (RNFL) has the potential to open a diagnostic window on monitoring neurodegeneration.

Here we present a systematic review of studies investigating OCT in patients with MS. Special care is taken to distinguish axonal damage caused by MS clinically evident optic neuritis (MSON) from more subtle retinal axonal damage in non-affected MS eyes. We will review the anatomical functional correlations, first focusing on the visual system and second, focusing on more global measures of disability in MS. The relationship of OCT data to established electrophysiological techniques and imaging modalities will also be discussed. Finally, we will provide a glimpse into future research areas of advanced OCT imaging that might influence assessment of axonal damage relevant to assessing a patient's MS activity and response to therapy. 


\section{Methods}

Search strategy and selection criteria The review of the Dutch, English, French, German, Italian and Spanish literature was conducted on all studies using OCT in MS patients between the first description of the method by Huang ${ }^{1}$ and May 2010, including manuscripts published ahead of print. We searched pubmed, EMBASE, medline, web of science and the Cochrane Register of Diagnostic Test Accuracy Studies using the search terms: multiple sclerosis, MS, optic neuritis, ON, optical coherence tomography, OCT, retinal nerve fibre layer, RNFL. From 99 studies identified, 62 were excluded because they were reviews, did not use the Stratus OCT, were single case reports, communications in response to an article, duplication of data already published on this cohort or data presentation were not detailed enough to allow inclusion into a meta-analysis. Studies that did not include a control cohort were only included if they compared the ON affected and not affected eye in MS patients. From the 37 included studies 35 presented data suitable for meta-analysis of RNFL thickness between groups. ${ }^{4-38}$

Statistical analysis The data analysis used the Cochrane Collaboration's Review Manager software package (RevMan5) following the guidance of the Diagnostic Test Accuracy (DTA) Working Group. The published RNFL thickness data was entered as a continuous variable. We used inverse variance, with random effects in the model. For effect measure we 
chose the mean difference which allows to compare the RNFL thickness in $\mu \mathrm{m}$ between the groups of interest. Three group comparisons for the RNFL thickness were performed: (a) MSON eyes with controls eyes, (b) MS eyes without history of MSON and control eyes, (c) in the same MS patient the MSON eye with the non-affected eye. Regression analyses were performed using SAS software (version 9.1.3). A p-value of $\leq 0.05$ was regarded as significant.

\section{OCT in Multiple Sclerosis}

Axonal loss in the retina With the invention of the ophthalmoscope (von Helmholtz, 1851), in vivo detection of atrophy of the optic disc became technically possible. For example, Gowers described sectoral RNFL loss in a woman suffering from syphilis. In his case the remaining nerve fibres were more visible because of swelling (plate II, figure 2 in reference ${ }^{39}$ ). A later case of sectoral RNFL loss in which sequential in vivo images permitted to evoke the concept of ascending axonal degeneration (now known as Wallerian degeneration) was facilitated by the rare presence of myelinated fibres in the retina. ${ }^{40}$ Bachmann describes a 28 year old man in whom sudden loss of vision in the right eye on the 22nd of January 1920 was due to a central retinal artery occlusion. Within two months after the retinal vascular event, the RNFL loss became visible as degeneration of bundles of myelinated retinal axons (Figure $2 \mathrm{~A}$ ), progressing over the following two months (Figure $2 \mathrm{~B}$ ) and leading to complete optic atrophy and 
loss of myelinated axons observed on fundus exam within one year (Figure $2 \mathrm{C}) .{ }^{40}$ There is good post-mortem evidence for RNFL thinning in MS. ${ }^{41}$

RNFL in MSON In multiple sclerosis, following optic neuritis (MSON), the degree of RNFL loss in thickness is in the range of $5-40 \mu \mathrm{m}$, averaging at 10-20 $\mu \mathrm{m} .{ }^{6}$ The finding was significant for all studies using time domain OCT (TD-OCT) technology based on the Stratus OCT (Zeiss, software version 3.0 \& 4.0) that we identified in a systematic literature review. The summary data of 14 studies on MS patients with MSON, 4, 10, 11, 16, 18, 19,21-27,30,31 containing a total of 2063 eyes tested with OCT are presented in Figure 3. From two of these studies the data published were not in a format allowing inclusion into the meta-analysis and more detailed information could not be obtained from the authors. ${ }^{24,28}$ The results of the meta-analysis on the remaining 12 studies ${ }^{4,10,11,16,18,21,22,25-27,30,31}$ are highly significant with an estimated average RNFL loss of $-20.38 \mu \mathrm{m}[-22.86,-17.91]$.

An important limitation of these studies is that optic nerve damage, independent of whether it occurs in the context of MS (MSON) or due to other causes (for a thorough clinical review see reference ${ }^{42}$ ) will cause some degree of RNFL loss. In an elegantly conducted small case series, Choi et al. present detailed OCT data on patients with several different kinds of optic neuropathy. ${ }^{43}$ Any form of optic nerve damage was associated with marked thinning of the RNFL. Therefore the question arises as to whether the presence of MSON could have introduced a bias towards more damage and RNFL thinning into the studies presented in Figure 3. 
In the absence of MSON, retrograde trans-synaptic retinal ganglion cell degeneration due to MS lesions within the posterior optic pathways could be a cause for RNFL loss. The existence of retrograde trans-synaptic retinal ganglion cell degeneration has been proven in patients with cerebrovascular accidents affecting the posterior visual pathways and cortex. ${ }^{44,45}$

In a combined MRI/OCT study Reich et al. showed that damage to the optic radiations in MS was associated with a reduced averaged global RNFL. ${ }^{46}$ There is a need to investigate whether the recently recognised effect of post geniculate lesions ${ }^{44,45}$ on the RNFL can be distinguished from RNFL loss caused by subclinical damage to the anterior visual pathways in MS as suggested by VEP studies. ${ }^{47-51}$

RNFL in MS without MSON We identified 16 studies which compared the RNFL thickness in patients with MS without evidence for ON to a control population, ${ }^{4,10,11,14-16,19,21,22,25-28,30,31}$ of which 15 provided data in a format allowing inclusion into the meta-analysis. $4,10,11,14,16,21,22,25-28,30,31$ The summary data on the 3154 eyes investigated is presented in Figure 4. The estimated RNFL loss $(-7.08 \mu \mathrm{m})$ was less compared to MSON $(-22.86 \mu \mathrm{m})$, but the $95 \% \mathrm{Cl}$ were markedly smaller [-8.65, -5.52]. This highlights the importance for carefully looking for evidence of MSON in order to minimise the risk that detection of the more subtle RNFL loss due to presumed retrograde trans-synaptic retinal ganglion cell degeneration may be masked by the pronounced loss caused by damage to the anterior visual pathways. 
RNFL in MS comparing MSON eyes with the contralateral eye To address this question 27 studies compared the affected (MSON) eye to the clinically non-affected eye in MS patients. ${ }^{4-6,8-11,13,16,17,21-25,27,29-38}$ The meta-analysis on the 4199 eyes investigated clearly illustrates the significant effect that presence of MSON has on RNFL (Figure 5). The estimate RNFL loss $(-13.84 \mu \mathrm{m})$ is larger than what was seen for the comparison of MS without MSON to controls $(-7.08 \mu \mathrm{m})$, but the confidence intervals were overlapping. An important prognostic finding of these studies is that after reaching a threshold of thinning to about $75 \mu \mathrm{m}$ of RNFL thickness, the chances for recovery of visual function seem to be less. ${ }^{6}$

Conclusion Taken together the published data $a^{4-27,29-38}$ suggests a relationship between RNFL thinning and MS pathology as illustrated in Figure 6 . The retinal axons project through the optic nerve into the lateral geniculate nucleus (LGN). About $90 \%$ of retinal axons synapse in the LGN and travel with the optic radiations to the occipital cortex. The remaining $10 \%$ project into the pretectal region of the midbrain. Severe thinning of the RNFL layers follows MSON directly (Figure $6 \mathrm{~B}$ ). These acute changes in MSON patients can be distinguished from more chronic changes caused by MS pathology of the optic pathways. An MS lesion affecting fibres in the optic radiation will cause Wallerian degeneration of some axons which reaches the LGN only after some time. Due to trans-synaptic degeneration retrograde axonal degeneration will eventually cause some degree of RNFL thinning (Figure $6 \mathrm{C}$ ). It is also likely that progressive loss of retinal 
ganglion cells (RGC) occurs as a result of chronic changes in the optic nerves/anterior visual pathways themselves (Figure $6 \mathrm{D}$ ). To test this hypothesis we reviewed the literature for the time course of RNFL loss in MS patients with and without MSON.

\section{Time course of RNFL loss in MS}

As a rule of thumb, RNFL loss becomes readily detectable to OCT about 3 months after an acute optic neuritis (ON). Earlier reduction in RNFL thickness from axonal atrophy is clinically difficult to distinguish from a reduction due to resolution of axonal swelling, which is common in acute optic neuritis. Costello et al. presented longitudinal data on the RNFL thickness during the first 12 months following ON (see Figure 7). This data shows ongoing axonal loss in the affected eye for at least 12 months, but most thinning occurs by 6 months after injury.

An inverse correlation between disease duration and the averaged overall RNFL thickness was found by some $R=-0.262, p=0.011,{ }^{19} R=-0.6, p=0.02,{ }^{25}$ $\mathrm{p}=0.03$ (R-value not published), ${ }^{10}$ but not by others. ${ }^{15,16}$ Examining nonMSON eyes Henderson et al. found that the RNFL decreased by $0.12 \mathrm{mum}$ per year of disease $(95 \% \mathrm{Cl}:-0.50,0.25)$, but this failed statistical significance $(p=0.513) .{ }^{15}$ Investigating MSON eyes, Klistoner et al. did not find a correlation between RNFL thickness and disease duration ( $p=0.9, R-$ value not published). ${ }^{16}$ The divergent results ${ }^{10,15,16,19,25}$ may in part be explained by the variation in average disease duration and a bias in the population of 
MS patients studied. A meta-regression analysis of the studies' raw data may help to shed light on the presumed association between RNFL loss and disease duration, but the most accurate information will come from longitudinal studies, where the RNFL thickness in individual patients can be studied over time. Talman et al. published longitudinal data from 593 eyes which were assessed at baseline and $\geq 6$ months later. ${ }^{38}$ Their statistical analysis was corrected for patients age and adjusted for within-patient and inter eye correlations. For MSON eyes the percentage decrease of RNFL thickness compared to baseline was $0.4 \%(0.4 \mu \mathrm{m} ; 95 \% \mathrm{Cl}: 1.16$, 0.35) for eyes with a $0.5-1$ year follow-up period; $1.7 \%(1.6 \mu \mathrm{m} ; 95 \% \mathrm{Cl}$, $2.47,0.70)$ for $>1$ to 2 years; $3.2 \%(2.9 \mu \mathrm{m} ; 95 \% \mathrm{Cl}, 4.02,1.86)$ for $>2$ to 3 years; and $6.7 \%(6.1 \mu \mathrm{m} ; 95 \% \mathrm{Cl}, 7.73,4.41)$ for $>3$ years of follow-up. In comparison the average RNFL thinning for disease-free control eyes was $0.5 \%(0.49 \mu \mathrm{m} ; 95 \% \mathrm{Cl}, 1.36,-0.39)$ over a 3 -year period. ${ }^{38}$ Their pooled analysis (MSON eyes and non-MSON eyes) showed that each year of follow-up was associated with an , on averaged $2 \mu \mathrm{m}$ increase of RNFL thinning ( $p<0.001$, generalised estimating equation models). ${ }^{38}$

Conclusion One needs to be very careful drawing conclusions on the time course of RNFL loss in an individual eye from cross-sectional data. The data suggest that in MS without MSON the estimated yearly thinning of the overall RNFL $\left(\approx 2 \mu \mathrm{m}^{38}\right)$ is probably below the detection limit of TD-OCT systems. Theoretically, the newer Spectral or Fourier domain OCT (SD/FD-OCT) systems should be able to enable resolution at this 
level, ${ }^{52-55}$ but practically a resolution of $4-6 \mu$ was found for the Heidelberg spectralis and Cirrus HD-OCT. ${ }^{56}$ There was (yet) no quantifiable RNFL loss in patients with a recent onset (average of 4.3 months) clinically isolated syndrome (CIS) if compared to healthy controls. ${ }^{57}$ The current duration of phase II trials in MS is frequently around 4-6 months and it is not likely that OCT will provide a reliable outcome over this time-scale. From the above data one would expect that MS patients without MSON will require follow-up for at least two years.

The longitudinal monitoring of the RNFL is technically challenging. Two methods have been validated clinically: topographic change analysis (TCA) and statistical image mapping (SIM) ${ }^{58-61}$ There is no consensus yet on how to collect and analyse longitudinally collected OCT data in MS.

\section{OCT and disability in MS}

There are well characterised limitations to the clinical disability scales currently employed in MS research, ${ }^{62}$ not least of which is that they fail to fully capture the range of disability seen in the disease, especially if they are not in the domain of patient mobility and motor function. Useful surrogate markers are challenging to validate.

The driving force of disability in MS, axonal loss, appears to be associated with changes in the RNFL which are statistically related to the changes observed in clinical disability progression. ${ }^{4-27}$ (Figures 3-5). Because the RNFL is anatomically related to visual function, studies analysing 
this relationship are reviewed first. There are good arguments that RNFL loss also reflects neurodegeneration on a more global level ${ }^{2,3}$ stimulating additional review of the robustness of the relationship between RNFL loss and global clinical disability scales. In reviewing this literature, it is important to keep in perspective that almost all of the studies to date are performed on group analysis.

\subsection{Visual function}

Visual acuity Monocular visual acuity (VA) is commonly assessed using standard eye charts. The method was introduced by the Dutch ophthalmologist Hermann Snellen in $1862 .^{63}$ The current convention is to document the Snellen equivalent (20/10-20/200). There are methodological limitations of the Snellen charts and of the many published improvements this review will consider Early Treatment Diabetic Retinopathy Study (ETDRS) charts and low contrast acuity (Sloan charts) in a separate paragraph.

Loss of RNFL was associated with reduced Snellen VA in most ${ }^{5,6,18,19,21,27,64-66}$ but not all ${ }^{13}$ studies. A linear correlation between loss of Snellen VA and the RNFL was observed in three studies. ${ }^{18,64,65}$ The strongest correlations $(\mathrm{R}>0.6)$ were found in studies including MSON patients. ${ }^{18,64,65}$ Comparable results were found for use of standard Japanese decimal VA. ${ }^{35}$

Early Treatment Diabetic Retinopathy Study (ETDRS) charts The ETDRS charts have a number of advantages over the Snellen VA, one being that the use of logarithmic scaling (logMAR), permits adjustment of the VA 
score to the viewing distance. ${ }^{67}$ Essentially a logMAR score provides interval data which facilitate statistical analyses and is therefore recommended for use in clinical trials. ${ }^{68}$ Trip et al. found a linear correlation between the interocular difference in the logMAR score and the RNFL thickness. ${ }^{26}$ Henderson et al confirmed the correlation of the RNFL with the logMAR score $(R=-0.54, p<0.001) .{ }^{15}$ Costello et al. also observed such a correlation six months after MSON. ${ }^{7}$ In a mixed cohort of untreated MS patients with or without MSON, Spain et al. reported a linear correlation $(R=-0.53$, $p<0.001)^{29}$ which is consistent with the results of Siepman et al. $(R=-0.56$, $\mathrm{p}<0.01) .{ }^{37}$

Low contrast letter acuity Balcer et al. first suggested integrating (binocular) low contrast letter acuity (Sloane charts) into a modified Multiple Sclerosis Functional Composite (MSFC) based on her observation that MS patients could be significantly $(p<0.0001)$ better distinguished from controls at a $1.25 \%$ contrast level compared to ETDRS charts. ${ }^{69}$ The authors also found Pelli-Robson charts to be of use, albeit at a lower level of significance $(p=0.003)$. The results were confirmed in later studies. ${ }^{38,70}$

Fisher et al. found a one-line change of $1.25 \%$ low-contrast letter acuity for every $4 \mu \mathrm{m}$ of RNFL lost. ${ }^{10}$ A strong correlation ( $\left.R=0.63, p=0.02\right)$ between low contrast acuity and RNFL thickness in affected MSON eyes was described in two other studies, ${ }^{11,27}$ a finding confirmed independently in patients with PPMS $(R=-0.46, p=0.026) .{ }^{15}$ A moderate correlation between the RNFL and $2.5 \%$ charts $(R=0.39, p<0.001)$ and $1.25 \%$ charts 
$(\mathrm{R}=0.31, \mathrm{p}<0.001)$ was found in one study. ${ }^{21}$ In line with these studies are the results of Spani et al. using 1.25\% Sloan charts $(R=-0.34, p=0.02){ }^{29}$ Currently, there are no longitudinal studies showing good correlation between RNFL and VA over time.

Visual fields (VF) Multiple sclerosis can produce any type of VF defect. The most common VF defects in acute MSON are dense but transient scotoma which are central, altitudinal or centro-caecal. ${ }^{42,71,72}$ Achromatic static perimetry is typically used for assessment of VF loss. The sensitivity of this technique is limited when supra-threshold screening strategies are used and improves with threshold estimation strategies. ${ }^{73,74}$ The likelihood to underestimate VF loss depends on the number of stimuli tested, stimulus size, the threshold loss and the control of eye-movements.

Costello et al. used the central 30-2 full threshold strategy (Humphrey) ${ }^{6}$ which is sensitive and gives a good overview. The authors found a relationship between the RNFL thickness and VF 3-6 months after ON. Below a threshold of $75 \mu \mathrm{m}$ RNFL loss, a linear correlation was found with the VF mean deviation (MD) in decibels $(\mathrm{dB}){ }^{*}$. Importantly, there was no recovery of visual function in these patients. A RNFL thickness of less then $75 \mu \mathrm{m}$ is considered to be a poor prognostic sign. ${ }^{7,22,23}$ The results of Costello et al. are consistent with those by Trip et al who described a linear correlation between the interocular difference in the RNFL thickness and VF MD us-

*Authors note: the VF MD is one of four global indices provided by the Humphrey perimeter. The MD gives the average of differences from normal expected value for the patients age. The MD is useful for detecting diffuse VF loss as it is the case in MSON. 
ing the same protocol. ${ }^{26} \mathrm{~A}$ weak correlation between the averaged RNFL thickness and the MD VF was also confirmed by others. ${ }^{15,19,37,64}$ Noval et al. described an association between RNFL loss and VF 1.5 and 3 months after MSON, but not any more after 6 months. ${ }^{64}$ The authors conclude that OCT may detect RNFL damage at a level which is below the sensitivity of automated static perimetry which is consistent with other studies. ${ }^{20}$

Cheng et al. used a VF severity score and found a better correlation for the RNFL with the overall VF loss compared to quadrant VF loss. ${ }^{5}$ The authors suggest this may be due to (a) diffuse instead of localised RNFL defects in MS, (b) a poor structure-to-function map, as reasons for the limited topographic correlations, ${ }^{5}$ and suboptimal image registration on TD-OCT in some patients offers an alternative explanation. Trip et al. also failed to show an association between a temporal VF defect and loss of RNFL thickness in the corresponding RNFL sector. ${ }^{26}$ They point out that the Humphrey 30-2 program tests far less points in the nasal and temporal sectors than in the superior and inferior sectors, potentially leading to greater noise due to reduced sampling.

\subsection{Global clinical scores}

EDSS Twelve studies examined the relationship between loss of RNFL and progression on the EDSS. ${ }^{4,9,10,12,13,18,24,25,28,37,65,75}$ An inverse correlation between the RNFL and the EDSS was observed in 6 studies ( $R=-$ $0.348,{ }^{24} \mathrm{R}=-0.7,{ }^{25} \mathrm{R}=-0.399,{ }^{75} \mathrm{R}=-0.30,{ }^{37} \mathrm{R}=-0.42$ MS patients without 
$\mathrm{MSON}^{4}$ and partial $\mathrm{R}=-0.35$ using the minimal RNFL thickness ${ }^{12}$ ). This data is consistent with analyses from two further studies describing a significant reduction of the RNFL with increasing EDSS percentiles. ${ }^{10,13}$ Four studies did not find a relationship between the RNFL and the EDSS. ${ }^{18,28,36,65}$ One of these studies included NMO and MS patients, ${ }^{18}$ another presented detailed data on both variables but did not include a correlation analysis. ${ }^{9}$

The differences are at least in part explained by the heterogeneity of the groups investigated (see Figures 3-4). The strongest correlation between the EDSS and the RNFL was found for MS eyes which were not affected by MSON. ${ }^{4,25}$ It is possible that the strong effect MSON has on RNFL thickness (Figure 3) masks more subtle changes caused by either asymptomatic axon damage or trans-synaptic axonal degeneration in non-affected MS eyes.

Therefore, future treatment trials using OCT as a secondary outcome measure for global disability will have to anticipate that predefined analyses on patients without MSON are likely to be of stronger statistical power than in patients with MSON and that alternative measures of disability scoring that better reflect generalised neurologic functioning, including cognition may need to be used which might better reflect diffuse axonal loss in the CNS.

The Multiple Sclerosis Severity Score (MSSS) The MSSS was developed to overcome the problem of changes in the EDSS with different disease durations when comparing groups. There was no correlation of the 
RNFL with the MSSS in one study including patients with MSON. ${ }^{65}$

\section{OCT and electrophysiology in MS}

OCT allows to study of the structural properties of the retina thus complimenting the many electrophysiological techniques aimed at functional assessment. Research has been performed to find out possible associations between the two domains. Recalling the simplified sketch from Figure 6 , one can formulate two key hypotheses to be tested: (1) is there a relationship between RNFL loss and VEP/PERG amplitude reduction (Figure 6 B)? (2) does VEP evidence for demyelination in the context of normal anterior visual pathways predict later development of RNFL loss with transynaptic retrograde degeneration (Figure $6 \mathrm{C}$ )?

\subsection{VEP}

Following demyelination of the optic nerve the latency of visual evoked potentials (VEP) are typically prolonged. This finding may persist for many years and is regarded as a sensitive but not specific test. In a longitudinal study Brusa et al. were first to note a small decline of the VEP amplitude in the non-affected eye of MS patients with ON, suggesting that this may be due to axonal loss. ${ }^{50}$ Advancing this concept, the same group demonstrated that reduction of RNFL thickness was indeed related to reduced VEP (whole-field VEP and central-field VEP), ${ }^{26}$ a finding confirmed by others. ${ }^{16,19,20,76}$ Klistorner et al. went on to show a high functional- 
topographic correlation between multifocal VEP and the RNFL (inferior VF, $R=0.84$; superior $V F, R=0.78$; central $V F, R=0.75 ; p<0.000$ for all correlations). ${ }^{77}$ Advances in multifocal VEP data analysis may render this technique more accessible. ${ }^{78}$ Some authors ${ }^{14,16,19,79}$ also found a correlation between the RNFL and VEP latencies.

The association between reduction in RNFL and VEP amplitudes lends further weight to the argument that demyelination related damage to the ON may cause either direct axon damage or may occur in the postgeniculate visual pathway, leading to retrograde axonal degeneration of the nonmyelinated axons in the retina (Figure $6 \mathrm{~B}$ ).

\subsection{ERG}

Using TD-OCT a correlation was found between the RNFL thickness and pattern ERG P50 latency as well as with the P50-P95 amplitude. ${ }^{79}$ It was suggested that this may be due to loss of ganglion cells following damage to the optic nerve. ${ }^{79}$ Others did not find a convincing relationship between the overall averaged RNFL and simple or multifocal ERG results, ${ }^{14}$ possibly because multifocal ERG samples mainly the photoreceptor and bipolar cell activation and not ganglion cells, therefore a correlation may not necessarily be expected. 


\section{OCT and imaging in MS}

It is beyond the scope of this review to adequately address the results of the body of literature on structural changes within the visual pathways assessed by brain imaging techniques and the relationship to OCT data. In brief, this relationship was investigated in a number of studies included in our systematic review. ${ }^{11-13,24,25,80,81}$ These authors focused on optic nerve imaging ${ }^{11,80,81}$ and full brain imaging techniques. ${ }^{11-13,24,25,46}$ The published data suggest correlations of the RNFL with brain atrophy measures $^{12,13,25}$ but a consensus has yet to emerge which atrophy measure is the most relevant, with grey matter atrophy remaining a hot topic. ${ }^{11}$ Other measures potentially to be considered are the normalised brain volume, ${ }^{11,24}$ the T2 lesion volume, ${ }^{11,24,25}$ the magnetisation transfer ratio, ${ }^{11,81}$ the fractional anisotropy and diffusion tensor imaging. ${ }^{11,82}$ Less promising seem to be the $T 1$ lesion volume ${ }^{11,13,25}$ and the mean parenchymal diffusivity. ${ }^{11,46}$ For a more in-depth review see references. ${ }^{?, 3}$

\section{Future developments}

OCT and the macula in MS Changes of macular volume, as well as inner and outer macular segments consistently show volume loss caused by loss of retinal ganglion cells (RGC). ${ }^{12,15,19-23,26,32,37,83-85}$ All found the macular volume to be reduced in patients with MS if compared to controls. Loss of macular volume was correlated with loss of RNFL in 4 stud- 
ies. ${ }^{15,26,80,84}$ Of note retinal thickness in the macula is made up of many RGCs (cones in the macula have a 1:1 correlation with RGCs where as in the periphery many rods have a 1000:1 relationship with RGCs). Therefore the macula provides a model to test hypotheses on primary neuronal cell death followed by axonal loss. In a recent editorial, ${ }^{86}$ Waxman asked three crucial questions: (1) what are the mechanisms involved in RGC apoptosis? (2) could this be following axonal pathology or dysfunction? (3) alternatively, could RGC apoptosis be an example of primary neuronal injury independent from axonal damage?

Polarisation-sensitive OCT (PS-OCT) Specific tissue properties can be further investigated by recording the polarisation state of back-scattered light. ${ }^{87,88}$ Polarisation-sensitive OCT (PS-OCT) yields depth-resolved information on any light polarisation changing properties of the sample related to tissue birefringence. ${ }^{87,89-91}$ Importantly, birefringence of the RNFL is related to the structure of dominant axonal filaments such as neurofilaments and microtubles. ${ }^{92}$ The birefringence of the RNFL induces a quantifiable degree of phase retardation..$^{89-91,93}$

The group of de Boer demonstrated that the birefringence of the RNFL is not constant, but varies by a factor of 3 around the optic nerve head, with higher values reported in the superior and inferior quadrants, and lower values in the nasal and temporal quadrant. ${ }^{93}$ This distinguishes the RNFL from other retinal structures which are either polarisation-preserving (e.g. photoreceptor layer) or polarisation scrambling/depolarising (e.g. retinal 
pigmented epithelium, RPE). ${ }^{91}$ Because changes to the axonal cytoskeleton such as neurofilament compactness, phosphorylation and stoichiometry can precede axonal loss, ${ }^{94,95}$ there may be a chance to detect early stages of axonal pathology in MS using PS-OCT. Experimentally it has already been shown that change in RNFL birefringence precedes RNFL loss. ${ }^{96}$

Fluorescence labelling Fluorescence labelling of a protein (annexin 5) which binds to a key component initiating apoptosis (phosphatidylserine) allowed for real-time in vivo monitoring of RGC apoptosis. ${ }^{97}$ The detection of apoptosing retinal cells (DARC) technique provides a promising surrogate outcome for neuroprotective treatment strategies in glaucoma, dementia and potentially also MS. ${ }^{98,99}$ Analogous, labelling of mitochondria may allow for in vivo testing of the "virtual hypoxia" hypothesis in MS. ${ }^{100}$

Retinal sector analysis This review did not include OCT data on sector analysis of the retina. It can be hypothesised that retinal axons of certain retinal sectors are more vulnerable then others in MS. Therefore, quantitative analysis of these sectors may allow for sensitive detection of axonal loss. However there is a large normal variation of the appearance of the optic disc influencing the RNFL thickness which ought to be controlled for. ${ }^{101,102}$ 
RNFL thickness \& reflectivity maps The accurate localisation of focal and peripheral loss of retinal axons is challenging using individual circular RNFL scans at the optic nerve head. One possible approach is the development of RNFL thickness maps. ${ }^{90}$ Because the loss of retinal axons in MS is more diffuse than the arcuate bundle loss seen in glaucoma it can be speculated that an integrative approach combining PS-OCT data with RNFL thickness maps may have the potential to predict the topography of RNFL loss in MS.

Retinal layer segmentation algorithms With the introduction of SD/FDOCT retinal layer image quality potentially allows for segmentation and quantification of individual layers. There is histological evidence that MS not only affects the RNFL but also cellular layers. ${ }^{41}$ Therefore new segmentation algorithms for quantitative analyses of individual retinal layers may enable us to better investigate progression of neurodegeneration in MS.

Doppler OCT and vascular changes in MS There is some evidence that vascular co-morbidity is a poor prognostic sign in MS. ${ }^{103}$ Changes of the retinal vasculature such as perivasculitis are recognised in MS. Perivasculitis leads to extravascular hyaline deposits, hence the descriptive name vascular sheathing. It is likely that these changes lead to a more rigid retinal vasculature and thus a quicker pulse propagation from the posterior (choriodal) to the anterior (retinal vasculature) circulation. ${ }^{17}$ 
This could be investigate by combining SD-OCT with Doppler velocimetry (SD-ODT). SD-ODT is non-invasive and allows for accurate topographic localisation of retinal blood vessels.

OCM and action potentials The influence that action potential propagation has on light properties has been recognised by Frank ${ }^{104}$ who cited his work together with Kornakova ${ }^{105}$ in 1947 . The field flourished over the next 30 years (reviewed in ${ }^{106}$ ). Using optical coherence microscopy (OCM) the structural assessment of an action potential became reality. ${ }^{107,108}$ At present only in vitro monitoring of action potential propagation is possible. Functional imaging of the human retina in vivo would be highly desirable to investigate whether axonal dysfunction may precede RGC and/or RNFL loss.

\section{Conclusion}

There is much excitement about OCT in MS research and it may be permitted to cite one of the first neurologists who made extensive use of the ophthalmoscope, Hughlings Jackson (1835-1911): "It is not too much to say that, without an extensive knowledge of ophthalmology, a methodological investigation of diseases of the nervous system is not merely difficult, but impossible." 109

In conclusion, OCT is a relatively new and promising technique for potential monitoring neuroprotective treatment trials in MS. First, there is a 
clear pathological correlate (axonal loss). Second, the analytical reproducibility is excellent. Third, the sensitivity to change is twice as high as the normal physiological changes during ageing and over a magnitude above the averaged changes seen after ON. Fourth, it is of high clinical relevance, correlating with clinical measures (loss of visual function) thus capturing a fundamental feature associated with disability progression. Fifth, there is data from brain imaging and electrophysiological studies which suggests that the integration of OCT into MS research may allow for a more accurate view of structure-function relationships in understanding the pathophysiology of this enigmatic disease. Finally, it is predictive of a clinical outcome (poor visual recovery). It is proposed to investigate the role OCT for future MS research within a concentrated, resource saving approach using an international workforce (OCTiMS). 


\section{Acknowledgement}

We would like to thanks the following authors for providing details on their published data needed for the meta-analysis: Philipp Albrecht MD, Luc Jeanjean MD and Fiona Costello MD. We are grateful for the comments of an anonymous referee.

AP has received consulting fees from Novartis.

RK: 2009-2012 1R01EY018853-01A2 NIH (NEI) "Focal Structure Relationships in Macular Layers from 3D Spectral OCT" Role: Co-PI (1.8 months/year). 2010-2015 2R01EY002115-34 NIH (NEI) "Studying models and mechanisms of optic nerve diseases"

AG: has research support from the National Institutes of Health and Howard Hughes Medical Institute. He has previously been funded by the National MS Society and American Academy of Neurology Foundation. He has received payment for services from Biogen-IDEC and Applied Clinical Intelligence for work on a clinical end-point adjudication committee related to clinical trials of Daclizumab and from Projects in Knowledge for editorial work on CME related publications. He has also received honoraria from Novartis Pharmaceuticals and PROCE CME.

JdB: has no funding from industry. Research funding and funding for equipment from government and non governmental foundations: $\mathrm{VICI}$ career award. Title: Minimally invasive optical diagnostics in medicine. 09NIG 03 (FOM). Title: Minimally invasive optical coherence tomography for diagnosis and staging of early lung cancer lesions. R21-RR023139 
(NIH), Title: High Resolution 3D Optical Coherence Phase Microscopy. NWO groot: A facility for laser based microscopy to study living, biological systems at the Laser Centre, VU University Amsterdam.

PV: honorarium from Heidelberg Engeniering.

SS: has received research grants from Biogen Idec and Bayer Schering Pharma and consulting fees from Bayer Schering Pharma, Merck Serono and Sanofi-Aventis.

PC: has received grants from Biogen-IDEC, Teva, Vertex, Bayer, Genentech, and Serono. He has received consulting fees from; Novartis, BiogenIDEC, Teva, Vertex, Novonordisk, Centacor, Serono, and Genentech.

CP: received an institutional grant from Novartis; consultancy fees from Actelion, Biogen Idec, Bayer Schering, TEVA, Merck-Serono, Novartis, Glaxo SK, UCB, Roche, Antisense Ther; expert testimony: Biogen,

CP, RK, AG, PV, SS and PC are sitting on the Novartis steering committee for a multi-center observational study: "A 3-year, open-label, multicenter, multi-cohort, parallel-group study to validate optical coherence tomography in patients with multiple sclerosis" and receive honoraria.

Author's contributions AP: conceived the idea for this review, performed the literature search, systematic review and meta-analysis. Wrote the first draft of the manuscript. JdB: revised the manuscript, performed an independent literature research and provided Figures 8 and 9A. SS: revised the manuscript. PV: revised the manuscript. RK: revised the manuscript and performed an independent literature research. AG: revised the manuscript. 
PC: revised the manuscript. CP: revised the manuscript. 


\section{References}

[1] D. Huang, E. A. Swanson, C. P. Lin, J. S. Schuman, W. G. Stinson, W. Chang, M. R. Hee, T. Flotte, K. Gregory, and C. A. Puliafito. Optical coherence tomography. Science, 254(5035):1178-1181, Nov 1991.

[2] Elliot Frohman, Fiona Costello, Robert Zivadinov, Olaf Stuve, Amy Conger, Heather Winslow, Anand Trip, Teresa Frohman, and Laura Balcer. Optical coherence tomography in multiple sclerosis. Lancet Neurol, 5(10):853-863, Oct 2006.

[3] Frederik Barkhof, Peter A Calabresi, David H Miller, and Stephen C Reingold. Imaging outcomes for neuroprotection and repair in multiple sclerosis trials. Nat Rev Neurol, 5(5):256-266, May 2009.

[4] Philipp Albrecht, Ruth Frhlich, Hans-Peter Hartung, Bernd C Kieseier, and Axel Methner. Optical coherence tomography measures axonal loss in multiple sclerosis independently of optic neuritis. $J$ Neurol, 254(11):1595-1596, Nov 2007.

[5] Han Cheng, Michal Laron, Jade S Schiffman, Rosa A Tang, and Laura J Frishman. The relationship between visual field and retinal nerve fiber layer measurements in patients with multiple sclerosis. Invest Ophthalmol Vis Sci, 48(12):5798-5805, Dec 2007. 
[6] Fiona Costello, Stuart Coupland, William Hodge, Gianni R Lorello, Jeannie Koroluk, Y. Irene Pan, Mark S Freedman, David H Zackon, and Randy H Kardon. Quantifying axonal loss after optic neuritis with optical coherence tomography. Ann Neurol, 59(6):963-969, Jun 2006.

[7] F. Costello, W. Hodge, Y. I. Pan, E. Eggenberger, S. Coupland, and R. H. Kardon. Tracking retinal nerve fiber layer loss after optic neuritis: a prospective study using optical coherence tomography. Mult Scler, 14(7):893-905, Aug 2008.

[8] Fiona Costello, William Hodge, Y. Irene Pan, Luanne Metz, and Randy $\mathrm{H}$ Kardon. Retinal nerve fiber layer and future risk of multiple sclerosis. Can J Neurol Sci, 35(4):482-487, Sep 2008.

[9] Fiona Costello, William Hodge, Y. Irene Pan, Mark Freedman, and Christine DeMeulemeester. Differences in retinal nerve fiber layer atrophy between multiple sclerosis subtypes. J Neurol Sci, 281(12):74-79, Jun 2009.

[10] Jennifer B Fisher, Dina A Jacobs, Clyde E Markowitz, Steven L Galetta, Nicholas J Volpe, M. Ligia Nano-Schiavi, Monika L Baier, Elliot M Frohman, Heather Winslow, Teresa C Frohman, Peter A Calabresi, Maureen G Maguire, Gary R Cutter, and Laura J Balcer. Relation of visual function to retinal nerve fiber layer thickness in multiple sclerosis. Ophthalmology, 113(2):324-332, Feb 2006. 
[11] Elliot M Frohman, Michael G Dwyer, Teresa Frohman, Jennifer L Cox, Amber Salter, Benjamin M Greenberg, Sara Hussein, Amy Conger, Peter Calabresi, Laura J Balcer, and Robert Zivadinov. Relationship of optic nerve and brain conventional and nonconventional mri measures and retinal nerve fiber layer thickness, as assessed by oct and gdx: a pilot study. J Neurol Sci, 282(12):96-105, Jul 2009.

[12] E. Gordon-Lipkin, B. Chodkowski, D. S. Reich, S. A. Smith, M. Pulicken, L. J. Balcer, E. M. Frohman, G. Cutter, and P. A. Calabresi. Retinal nerve fiber layer is associated with brain atrophy in multiple sclerosis. Neurology, 69(16):1603-1609, Oct 2007.

[13] Erica Grazioli, Robert Zivadinov, Bianca Weinstock-Guttman, Norah Lincoff, Monika Baier, Jan Rang Wong, Sara Hussein, Jennifer L Cox, David Hojnacki, and Murali Ramanathan. Retinal nerve fiber layer thickness is associated with brain mri outcomes in multiple sclerosis. J Neurol Sci, 268(1-2):12-17, May 2008.

[14] Fatih C Gundogan, Seref Demirkaya, and Gungor Sobaci. Is optical coherence tomography really a new biomarker candidate in multiple sclerosis?-a structural and functional evaluation. Invest Ophthalmol Vis Sci, 48(12):5773-5781, Dec 2007.

[15] Andrew P D Henderson, S. Anand Trip, Patricio G Schlottmann, Daniel R Altmann, David F Garway-Heath, Gordon T Plant, and 
David $\mathrm{H}$ Miller. An investigation of the retinal nerve fibre layer in progressive multiple sclerosis using optical coherence tomography. Brain, 131(Pt 1):277-287, Jan 2008.

[16] Alexander Klistorner, Hemamalini Arvind, Than Nguyen, Raymond Garrick, Mark Paine, Stuart Graham, Justin O’Day, John Grigg, Francis Billson, and Con Yiannikas. Axonal loss and myelin in early on loss in postacute optic neuritis. Ann Neurol, 64(3):325-331, Sep 2008.

[17] A. Kochkorov, K. Gugleta, D. Kavroulaki, R. Katamay, K. Weier, M. Mehling, L. Kappos, J. Flammer, and S. Orgl. Rigidity of retinal vessels in patients with multiple sclerosis. Klin Monatsbl Augenheilkd, 226(4):276-279, Apr 2009.

[18] Harold Merle, Stphane Olindo, Anglique Donnio, Raymond Richer, Didier Smadja, and Philippe Cabre. Retinal peripapillary nerve fiber layer thickness in neuromyelitis optica. Invest Ophthalmol Vis Sci, 49(10):4412-4417, Oct 2008.

[19] V. Pueyo, J. Martin, J. Fernandez, C. Almarcegui, J. Ara, C. Egea, L. Pablo, and F. Honrubia. Axonal loss in the retinal nerve fiber layer in patients with multiple sclerosis. Mult Scler, 14(5):609-614, Jun 2008.

[20] Victoria Pueyo, Jose Ramon Ara, Carmen Almarcegui, Jesus Martin, Noemi Gerri, Elena Garca, Luis Emilio Pablo, Francisco Manuel 
Honrubia, and Francisco Javier Fernandez. Sub-clinical atrophy of the retinal nerve fibre layer in multiple sclerosis. Acta Ophthalmol, pages 1-5, Jun 2009.

[21] M. Pulicken, E. Gordon-Lipkin, L. J. Balcer, E. Frohman, G. Cutter, and P. A. Calabresi. Optical coherence tomography and disease subtype in multiple sclerosis. Neurology, 69(22):2085-2092, Nov 2007.

[22] J. N. Ratchford, M. E. Quigg, A. Conger, T. Frohman, E. Frohman, L. J. Balcer, P. A. Calabresi, and D. A. Kerr. Optical coherence tomography helps differentiate neuromyelitis optica and ms optic neuropathies. Neurology, 73(4):302-308, Jul 2009.

[23] A. R. Salter, A. Conger, T. C. Frohman, R. Zivadinov, E. Eggenberger, P. Calabresi, G. Cutter, L. Balcer, and E. M. Frohman. Retinal architecture predicts pupillary reflex metrics in ms. Mult Scler, 15(4):479-486, Apr 2009.

[24] Jorge Sepulcre, Manuel Murie-Fernandez, Angel Salinas-Alaman, Alfredo Garca-Layana, Bartolome Bejarano, and Pablo Villoslada. Diagnostic accuracy of retinal abnormalities in predicting disease activity in ms. Neurology, 68(18):1488-1494, May 2007.

[25] Malgorzata Siger, Krzysztof Dziegielewski, Lukasz Jasek, Marek Bieniek, Agnieszka Nicpan, Jerzy Nawrocki, and Krzysztof Selmaj. 
Optical coherence tomography in multiple sclerosis: thickness of the retinal nerve fiber layer as a potential measure of axonal loss and brain atrophy. J Neurol, 255(10):1555-1560, Oct 2008.

[26] S. Anand Trip, Patricio G Schlottmann, Stephen J Jones, Daniel R Altmann, David F Garway-Heath, Alan J Thompson, Gordon T Plant, and David H Miller. Retinal nerve fiber layer axonal loss and visual dysfunction in optic neuritis. Ann Neurol, 58(3):383-391, Sep 2005.

[27] Maulik S Zaveri, Amy Conger, Amber Salter, Teresa C Frohman, Steven L Galetta, Clyde E Markowitz, Dina A Jacobs, Gary R Cutter, Gui-Shuang Ying, Maureen G Maguire, Peter A Calabresi, Laura J Balcer, and Elliot M Frohman. Retinal imaging by laser polarimetry and optical coherence tomography evidence of axonal degeneration in multiple sclerosis. Arch Neurol, 65(7):924-928, Jul 2008.

[28] L. Jeanjean, G. Castelnovo, B. Carlander, M. Villain, F. Mura, G. Dupeyron, and P. Labauge. [retinal atrophy using optical coherence tomography (oct) in 15 patients with multiple sclerosis and comparison with healthy subjects]. Rev Neurol (Paris), 164(11):927934, Nov 2008.

[29] Rebecca I Spain, Mitchell Maltenfort, Robert C Sergott, and Thomas $\mathrm{P}$ Leist. Thickness of retinal nerve fiber layer correlates with disease duration in parallel with corticospinal tract dysfunction 
in untreated multiple sclerosis. J Rehabil Res Dev, 46(5):633-642, 2009.

[30] Amanda Quelly, Han Cheng, Michal Laron, Jade S Schiffman, and Rosa A Tang. Comparison of optical coherence tomography and scanning laser polarimetry measurements in patients with multiple sclerosis. Optom Vis Sci, May 2010.

[31] Markus Bock, Alexander U Brandt, Jan Drr, Helga Kraft, Nicholetta Weinges-Evers, Gunnar Gaede, Caspar F Pfueller, Katja Herges, Helena Radbruch, Stephanie Ohlraun, Judith Bellmann-Strobl, Jrn Kuchenbecker, Frauke Zipp, and Friedemann Paul. Patterns of retinal nerve fiber layer loss in multiple sclerosis patients with or without optic neuritis and glaucoma patients. Clin Neurol Neurosurg, May 2010.

[32] Elena Garcia-Martin, Victoria Pueyo, Jesus Martin, Carmen Almarcegui, Jose R Ara, Isabel Dolz, Francisco M Honrubia, and Francisco J Fernandez. Progressive changes in the retinal nerve fiber layer in patients with multiple sclerosis. Eur J Ophthalmol, 20(1):167-173, 2010.

[33] Michal Laron, Han Cheng, Bin Zhang, Jade S Schiffman, Rosa A Tang, and Laura J Frishman. Comparison of multifocal visual evoked potential, standard automated perimetry and optical coherence to- 
mography in assessing visual pathway in multiple sclerosis patients. Mult Scler, 16(4):412-426, Apr 2010.

[34] Harold Merle, Stephane Olindo, Angelique Donnio, Laurence Beral, Raymond Richer, Didier Smadja, and Philippe Cabre. Retinal nerve fiber layer thickness and spatial and temporal contrast sensitivity in multiple sclerosis. Eur J Ophthalmol, 20(1):158-166, 2010.

[35] Masahiko Nakamura, Toru Nakazawa, Hiroshi Doi, Takehiro Hariya, Kazuko Omodaka, Tatsuro Misu, Toshiyuki Takahashi, Kazuo Fujihara, and Kohji Nishida. Early high-dose intravenous methylprednisolone is effective in preserving retinal nerve fiber layer thickness in patients with neuromyelitis optica. Graefes Arch Clin Exp Ophthalmol, Mar 2010.

[36] C. Oreja-Guevara, S. Noval, B. Manzano, and E. Diez-Tejedor. Optic neuritis, multiple sclerosis-related or not: structural and functional study. Neurologia, 25(2):78-82, Mar 2010.

[37] Theodora A M Siepman, Marijke Wefers Bettink-Remeijer, and Rogier Q Hintzen. Retinal nerve fiber layer thickness in subgroups of multiple sclerosis, measured by optical coherence tomography and scanning laser polarimetry. J Neurol, May 2010.

[38] Lauren S Talman, Esther R Bisker, David J Sackel, David A Long, Kristin M Galetta, John N Ratchford, Deacon J Lile, Sheena K Farrell, Michael J Loguidice, Gina Remington, Amy Conger, Teresa C 
Frohman, Dina A Jacobs, Clyde E Markowitz, Gary R Cutter, GuiShuang Ying, Yang Dai, Maureen G Maguire, Steven L Galetta, Elliot M Frohman, Peter A Calabresi, and Laura J Balcer. Longitudinal study of vision and retinal nerve fiber layer thickness in multiple sclerosis. Ann Neurol, 67(6):749-760, Jun 2010.

[39] WR Gowers. A manual and atlas of medical ophthalmoscopy. London, Churchill, 1879.

[40] R Bachmann. Schwund markhaltiger Nervenfasern in der Netzhaut nach Embolie der Art. centralis retinae. v. Graefe's Arch. f. Ophth., 107:10-14, 1921.

[41] Ari J Green, Stephen McQuaid, Stephen L Hauser, Ingrid V Allen, and Roy Lyness. Ocular pathology in multiple sclerosis: retinal atrophy and inflammation irrespective of disease duration. Brain, Apr 2010.

[42] SJ Hickman, CM Dalton, DH Miller, and GT Plant. Management of acute optic neuritis. Lancet, 360:1953-1962, 2002.

[43] Stacey S Choi, Robert J Zawadzki, John L Keltner, and John S Werner. Changes in cellular structures revealed by ultra-high resolution retinal imaging in optic neuropathies. Invest Ophthalmol Vis Sci, 49(5):2103-2119, May 2008. 
[44] Jodhbir S Mehta and Gordon T Plant. Optical coherence tomography (oct) findings in congenital/long-standing homonymous hemianopia. Am J Ophthalmol, 140(4):727-729, Oct 2005.

[45] P. Jindahra, A. Petrie, and G. T. Plant. Retrograde trans-synaptic retinal ganglion cell loss identified by optical coherence tomography. Brain, 132:628-634, 2009.

[46] Daniel S Reich, Seth A Smith, Eliza M Gordon-Lipkin, Arzu Ozturk, Brian S Caffo, Laura J Balcer, and Peter A Calabresi. Damage to the optic radiation in multiple sclerosis is associated with retinal injury and visual disability. Arch Neurol, 66(8):998-1006, Aug 2009.

[47] K. H. Chiappa and A. H. Ropper. Evoked potentials in clinical medicine (second of two parts). N Engl J Med, 306(20):1205-1211, May 1982.

[48] AM Halliday, editor. Evoked potentials in clinical testing. Churchill Livingstone, London, 2nd edition, 1993.

[49] A. Brusa, S. J. Jones, R. Kapoor, D. H. Miller, and G. T. Plant. Longterm recovery and fellow eye deterioration after optic neuritis, determined by serial visual evoked potentials. J Neurol, 246(9):776-782, Sep 1999. 
[50] A. Brusa, S. J. Jones, and G. T. Plant. Long-term remyelination after optic neuritis: A 2-year visual evoked potential and psychophysical serial study. Brain, 124(Pt 3):468-479, Mar 2001.

[51] P Fuhr, A Borggrefe-Chappuis, C Schindler, and L Kappos. Visual and motor evoked potentials in the course of multiple sclerosis. Brain, 124:2162-2108, 2001.

[52] Maciej Wojtkowski, Vivek Srinivasan, James G Fujimoto, Tony Ko, Joel S Schuman, Andrzej Kowalczyk, and Jay S Duker. Threedimensional retinal imaging with high-speed ultrahigh-resolution optical coherence tomography. Ophthalmology, 112(10):1734-1746, Oct 2005.

[53] Teresa C Chen, Audrey Zeng, Wei Sun, Mircea Mujat, and Johannes $\mathrm{F}$ de Boer. Spectral domain optical coherence tomography and glaucoma. Int Ophthalmol Clin, 48(4):29-45, 2008.

[54] Barry Cense, Nader Nassif, Teresa Chen, Mark Pierce, SeokHyun Yun, B. Park, Brett Bouma, Guillermo Tearney, and Johannes de Boer. Ultrahigh-resolution high-speed retinal imaging using spectral-domain optical coherence tomography. Opt Express, 12(11):2435-2447, May 2004.

[55] Maciej Wojtkowski, Vivek Srinivasan, Tony Ko, James Fujimoto, Andrzej Kowalczyk, and Jay Duker. Ultrahigh-resolution, high-speed, 
fourier domain optical coherence tomography and methods for dispersion compensation. Opt Express, 12(11):2404-2422, May 2004.

[56] Ute E K Wolf-Schnurrbusch, Lala Ceklic, Christian K Brinkmann, Milko E lliev, Manuel Frey, Simon P Rothenbuehler, Volker Enzmann, and Sebastian Wolf. Macular thickness measurements in healthy eyes using six different optical coherence tomography instruments. Invest Ophthalmol Vis Sci, 50(7):3432-3437, Jul 2009.

[57] Olivier Outteryck, Hlne Zephir, Sabine Defoort, Marie Bouyon, Philippe Debruyne, Ikram Bouacha, Didier Ferriby, Arnaud Lacour, Pierre Labalette, Jerome de Seze, and Patrick Vermersch. Optical coherence tomography in clinically isolated syndrome: no evidence of subclinical retinal axonal loss. Arch Neurol, 66(11):1373-1377, Nov 2009.

[58] Frederick S Mikelberg. Assessment of the optic disc to measure neuroprotection. Can J Ophthalmol, 42(3):421-424, Jun 2007.

[59] B. C. Chauhan, J. W. Blanchard, D. C. Hamilton, and R. P. LeBlanc. Technique for detecting serial topographic changes in the optic disc and peripapillary retina using scanning laser tomography. Invest Ophthalmol Vis Sci, 41(3):775-782, Mar 2000.

[60] B. C. Chauhan, T. A. McCormick, M. T. Nicolela, and R. P. LeBlanc. Optic disc and visual field changes in a prospective longitudinal 
study of patients with glaucoma: comparison of scanning laser tomography with conventional perimetry and optic disc photography. Arch Ophthalmol, 119(10):1492-1499, Oct 2001.

[61] Andrew J Patterson, David F Garway-Heath, Nicholas G Strouthidis, and David P Crabb. A new statistical approach for quantifying change in series of retinal and optic nerve head topography images. Invest Ophthalmol Vis Sci, 46(5):1659-1667, May 2005.

[62] R. A. Rudick, C. H. Polman, J. A. Cohen, M. K. Walton, A. E. Miller, C. Confavreux, F. D. Lublin, M. Hutchinson, P. W. O'Connor, S. R. Schwid, L. J. Balcer, F. Lynn, M. A. Panzara, and A. W. Sandrock. Assessing disability progression with the multiple sclerosis functional composite. Mult Scler, 15(8):984-997, Aug 2009.

[63] H Snellen and E Landolt. Handbuch der gesamten Augenheilkunde, chapter Die Funktionsprüfung des Auges, pages 1-248. Engelman, Leipzig, Germany, 1874.

[64] Susana Noval, Ins Contreras, Gema Rebolleda, and Francisco J Muoz-Negrete. Optical coherence tomography versus automated perimetry for follow-up of optic neuritis. Acta Ophthalmol Scand, 84(6):790-794, Dec 2006.

[65] R. T. Naismith, N. T. Tutlam, J. Xu, J. B. Shepherd, E. C. Klawiter, S-K. Song, and A. H. Cross. Optical coherence tomography is less 
sensitive than visual evoked potentials in optic neuritis. Neurology, 73(1):46-52, Jul 2009.

[66] R. T. Naismith, N. T. Tutlam, J. Xu, E. C. Klawiter, J. Shepherd, K. Trinkaus, S-K. Song, and A. H. Cross. Optical coherence tomography differs in neuromyelitis optica compared with multiple sclerosis. Neurology, 72(12):1077-1082, Mar 2009.

[67] I. L. Bailey and J. E. Lovie. New design principles for visual acuity letter charts. Am J Optom Physiol Opt, 53(11):740-745, Nov 1976.

[68] [no authors listed]. Recommended stardard procedures for the clinical measurement and specification of visual acuity. report of working group 39. committee on vision. assembly of behavioral and social sciences, national research council, national academy of sciences, washington, d.c. Adv Ophthalmol, 41:103-148, 1980.

[69] L. J. Balcer, M. L. Baier, J. A. Cohen, M. F. Kooijmans, A. W. Sandrock, M. L. Nano-Schiavi, D. C. Pfohl, M. Mills, J. Bowen, C. Ford, F. R. Heidenreich, D. A. Jacobs, C. E. Markowitz, W. H. Stuart, G-S. Ying, S. L. Galetta, M. G. Maguire, and G. R. Cutter. Contrast letter acuity as a visual component for the multiple sclerosis functional composite. Neurology, 61(10):1367-1373, Nov 2003.

[70] M. L. Baier, G. R. Cutter, R. A. Rudick, D. Miller, J. A. Cohen, B. Weinstock-Guttman, M. Mass, and L. J. Balcer. Low-contrast let- 
ter acuity testing captures visual dysfunction in patients with multiple sclerosis. Neurology, 64(6):992-995, Mar 2005.

[71] J Gerling, JH Meyer, and G Kommerell. Visual field defects in optic neuritis and anterior ischemic optic neuropathy: distinctive features. Graefes Arch Clin Exp Ophthalmol, 236:188-92, 1998.

[72] GT Plant and RF Hess. Regional threshold contrast sensitivity within the central visual field in optic neuritis. Brain, 110 ( Pt 2):489-515, 1987.

[73] A Petzold and GT Plant. Failure to detect bitemporal field defects due to chiasmal compression on a screening perimetry protocol. Neuro Ophthalmol, 24:357-361, 2001.

[74] Ulrich Schiefer, John P Pascual, Beth Edmunds, Elisabeth Feudner, Esther M Hoffmann, Chris A Johnson, Wolf A Lagrze, Norbert Pfeiffer, Pamela A Sample, Flemming Staubach, Richard G Weleber, Reinhard Vonthein, Elke Krapp, and Jens Paetzold. Comparison of the new perimetric gate strategy with conventional full-threshold and sita standard strategies. Invest Ophthalmol Vis Sci, 50(1):488-494, Jan 2009.

[75] J. Toledo, J. Sepulcre, A. Salinas-Alaman, A. Garca-Layana, M. Murie-Fernandez, B. Bejarano, and P. Villoslada. Retinal nerve fiber layer atrophy is associated with physical and cognitive disability in multiple sclerosis. Mult Scler, 14(7):906-912, Aug 2008. 
[76] M. J. Thurtell, E. Bala, S. S. Yaniglos, J. C. Rucker, N. S. Peachey, and R. J. Leigh. Evaluation of optic neuropathy in multiple sclerosis using low-contrast visual evoked potentials. Neurology, 73(22):1849-1857, Dec 2009.

[77] Alexander Klistorner, H. Arvind, T. Nguyen, R. Garrick, M. Paine, S. Graham, J. O'Day, and C. Yiannikas. Multifocal vep and oct in optic neuritis: a topographical study of the structure-function relationship. Doc Ophthalmol, 118(2):129-137, Apr 2009.

[78] Donald C Hood, Jeffrey G Odel, and Bryan J Winn. The multifocal visual evoked potential. J Neuroophthalmol, 23(4):279-289, Dec 2003.

[79] V. Parisi, G. Manni, M. Spadaro, G. Colacino, R. Restuccia, S. Marchi, M. G. Bucci, and F. Pierelli. Correlation between morphological and functional retinal impairment in multiple sclerosis patients. Invest Ophthalmol Vis Sci, 40(11):2520-2527, Oct 1999.

[80] S. Anand Trip, Patricio G Schlottmann, Stephen J Jones, Wai-Yung Li, David F Garway-Heath, Alan J Thompson, Gordon T Plant, and David H Miller. Optic nerve atrophy and retinal nerve fibre layer thinning following optic neuritis: evidence that axonal loss is a substrate of mri-detected atrophy. Neuroimage, 31(1):286-293, May 2006.

[81] S. A. Trip, P. G. Schlottmann, S. J. Jones, W-Y. Li, D. F. GarwayHeath, A. J. Thompson, G. T. Plant, and D. H. Miller. Optic nerve 
magnetization transfer imaging and measures of axonal loss and demyelination in optic neuritis. Mult Scler, 13(7):875-879, Aug 2007.

[82] R. T. Naismith, J. Xu, N. T. Tutlam, A. Snyder, T. Benzinger, J. Shimony, J. Shepherd, K. Trinkaus, A. H. Cross, and S-K. Song. Disability in optic neuritis correlates with diffusion tensor-derived directional diffusivities. Neurology, 72(7):589-594, Feb 2009.

[83] C. Almarcegui, I. Dolz, V. Pueyo, E. Garcia, F. J. Fernandez, J. Martin, J. R. Ara, and F. Honrubia. Correlation between functional and structural assessments of the optic nerve and retina in multiple sclerosis patients. Neurophysiol Clin, 40(3):129-135, Jun 2010.

[84] Bryn M Burkholder, Benjamin Osborne, Michael J Loguidice, Esther Bisker, Teresa C Frohman, Amy Conger, John N Ratchford, Christina Warner, Clyde E Markowitz, Dina A Jacobs, Steven L Galetta, Gary R Cutter, Maureen G Maguire, Peter A Calabresi, Laura J Balcer, and Elliot M Frohman. Macular volume determined by optical coherence tomography as a measure of neuronal loss in multiple sclerosis. Arch Neurol, 66(11):1366-1372, Nov 2009.

[85] Andrew P D Henderson, S. A. Trip, P. G. Schlottmann, D. R. Altmann, D. F. Garway-Heath, G. T. Plant, and D. H. Miller. A preliminary longitudinal study of the retinal nerve fiber layer in progressive multiple sclerosis. J Neurol, Feb 2010. 
[86] Stephen G Waxman and Joel A Black. Retinal involvement in multiple sclerosis. Neurology, 69(16):1562-1563, Oct 2007.

[87] J. F. de Boer, T. E. Milner, M. J. van Gemert, and J. S. Nelson. Two-dimensional birefringence imaging in biological tissue by polarization-sensitive optical coherence tomography. Opt Lett, 22(12):934-936, Jun 1997.

[88] J. F. de Boer, T. E. Milner, and J. S. Nelson. Determination of the depth-resolved stokes parameters of light backscattered from turbid media by use of polarization-sensitive optical coherence tomography. Opt Lett, 24(5):300-302, Mar 1999.

[89] Barry Cense, Teresa C Chen, B. Hyle Park, Mark C Pierce, and Johannes $\mathrm{F}$ de Boer. Invivo depth-resolved birefringence measurements of the human retinal nerve fiber layer by polarization-sensitive optical coherence tomography. Opt Lett, 27(18):1610-1612, Sep 2002.

[90] Mircea Mujat, B. Hyle Park, Barry Cense, Teresa C Chen, and Johannes $\mathrm{F}$ de Boer. Autocalibration of spectral-domain optical coherence tomography spectrometers for in vivo quantitative retinal nerve fiber layer birefringence determination. J Biomed Opt, 12(4):041205, 2007.

[91] E. Gtzinger, M. Pircher, B. Baumann, C. Hirn, C. Vass, and C. K. Hitzenberger. Retinal nerve fiber layer birefringence evaluated with 
polarization sensitive spectral domain oct and scanning laser polarimetry: a comparison. J Biophotonics, 1(2):129-139, May 2008.

[92] Xiang-Run Huang and Robert W Knighton. Microtubules contribute to the birefringence of the retinal nerve fiber layer. Invest Ophthalmol Vis Sci, 46(12):4588-4593, Dec 2005.

[93] Barry Cense, Teresa C Chen, B. Hyle Park, Mark C Pierce, and Johannes $\mathrm{F}$ de Boer. Thickness and birefringence of healthy retinal nerve fiber layer tissue measured with polarization-sensitive optical coherence tomography. Invest Ophthalmol Vis Sci, 45(8):26062612, Aug 2004.

[94] A. Petzold, D. Gveric, M. Groves, K. Schmierer, D. Grant, M. Chapman, G. Keir, L. Cuzner, and E. J. Thompson. Phosphorylation and compactness of neurofilaments in multiple sclerosis: indicators of axonal pathology. Exp Neurol, 213:326-335, 2008.

[95] Axel Petzold, Edward J Thompson, Geoffrey Keir, Niall Quinn, Bjorn Holmberg, Nil Dizdar, Gregor K Wenning, Olivier Rascol, Eduardo Tolosa, and Lars Rosengren. Longitudinal one-year study of levels and stoichiometry of neurofilament heavy and light chain concentrations in csf in patients with multiple system atrophy. J Neurol Sci, 279(1-2):76-79, Apr 2009.

[96] Brad Fortune, Grant A Cull, and Claude F Burgoyne. Relative course of retinal nerve fiber layer birefringence and thickness and retinal 
function changes after optic nerve transection. Invest Ophthalmol Vis Sci, 49(10):4444-4452, Oct 2008.

[97] M. Francesca Cordeiro, Li Guo, Vy Luong, Glen Harding, Wei Wang, Helen E Jones, Stephen E Moss, Adam M Sillito, and Frederick W Fitzke. Real-time imaging of single nerve cell apoptosis in retinal neurodegeneration. Proc Natl Acad Sci U S A, 101(36):1335213356, Sep 2004.

[98] L. Guo, J. Duggan, and M. F. Cordeiro. Alzheimer's disease and retinal neurodegeneration. Curr Alzheimer Res, 7(1):3-14, Feb 2010.

[99] Li Guo and M. Francesca Cordeiro. Assessment of neuroprotection in the retina with darc. Prog Brain Res, 173:437-450, 2008.

[100] Bruce D Trapp and Peter K Stys. Virtual hypoxia and chronic necrosis of demyelinated axons in multiple sclerosis. Lancet Neurol, 8(3):280-291, Mar 2009.

[101] Donald L Budenz, Douglas R Anderson, Rohit Varma, Joel Schuman, Louis Cantor, Jonathan Savell, David S Greenfield, Vincent Michael Patella, Harry A Quigley, and James Tielsch. Determinants of normal retinal nerve fiber layer thickness measured by stratus oct. Ophthalmology, 114(6):1046-1052, Jun 2007.

[102] Choul Yong Park, Yun Taek Kim, and Changwon Kee. Evaluation of the influence of tilt of optic disc on the measurement of optic disc 
variables obtained by optical coherence tomography and confocal scanning laser ophthalmoscopy. J Glaucoma, 14(3):210-214, Jun 2005.

[103] R. A. Marrie, R. Horwitz, G. Cutter, T. Tyry, D. Campagnolo, and T. Vollmer. Comorbidity delays diagnosis and increases disability at diagnosis in ms. Neurology, 72(2):117-124, Jan 2009.

[104] G.M. Frank. Physical, chemical and structural processes during initiation and propagation of impulses through the nerve firbre. $I z v$. Akad. Nauk. SSSR, Ser. Biol., 1:26-38, 1947. [Article in Russian].

[105] YE.V. Kornakova, G.M. Frank, and L.N. Shteyngauz. Structural processes in the nerve. Fiziol. J. USSR, 33:483, 1947. [Article in Russian].

[106] L. B. Cohen. Changes in neuron structure during action potential propagation and synaptic transmission. Physiol Rev, 53(2):373418, Apr 1973.

[107] Benedikt W Graf, Tyler S Ralston, Han-Jo Ko, and Stephen A Boppart. Detecting intrinsic scattering changes correlated to neuron action potentials using optical coherence imaging. Opt Express, 17(16):13447-13457, Aug 2009. 
[108] Taner Akkin, Chulmin Joo, and Johannes F de Boer. Depth-resolved measurement of transient structural changes during action potential propagation. Biophys J, 93(4):1347-1353, Aug 2007.

[109] J Hughlings Jackson. Ophthalmology in its relation to general medicine. BMJ, 1:575-577, 1877. 


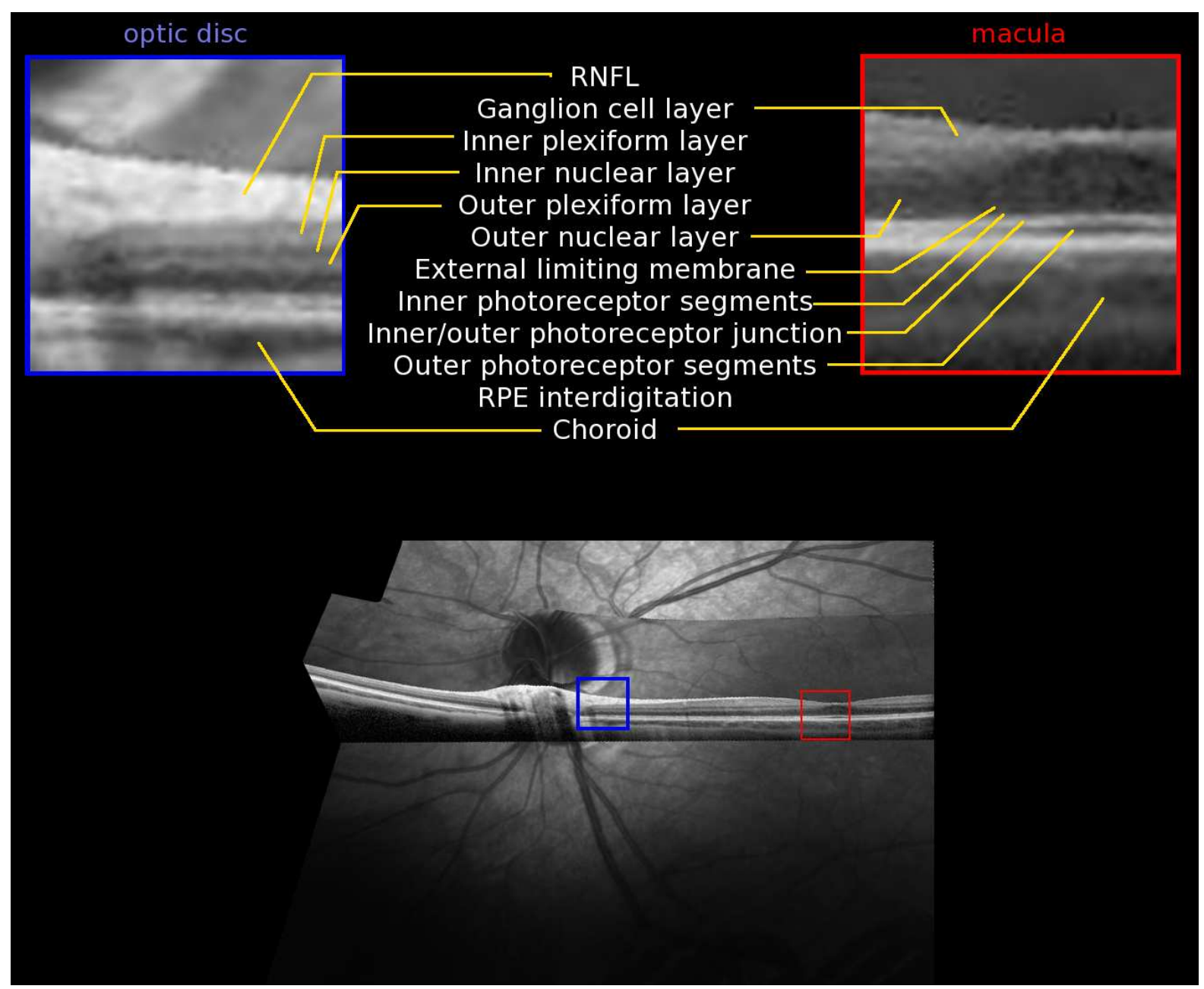

Figure 1: SD/FD-OCT image of a normal eye showing the different retinal layers. The inlay shows an enlarged part of the optic disc (blue frame) and macula (red frame). 
(A)

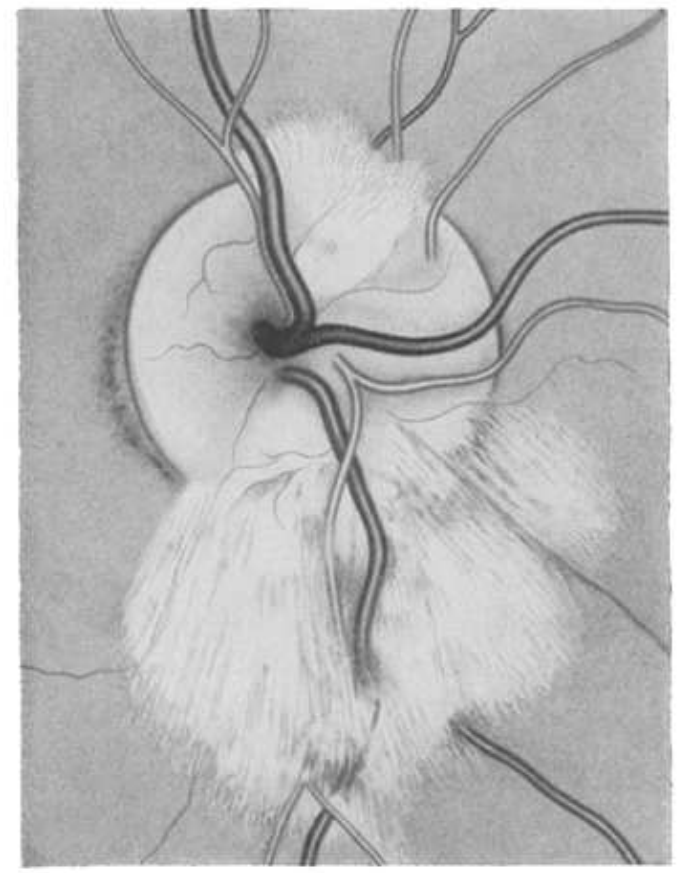

(B)

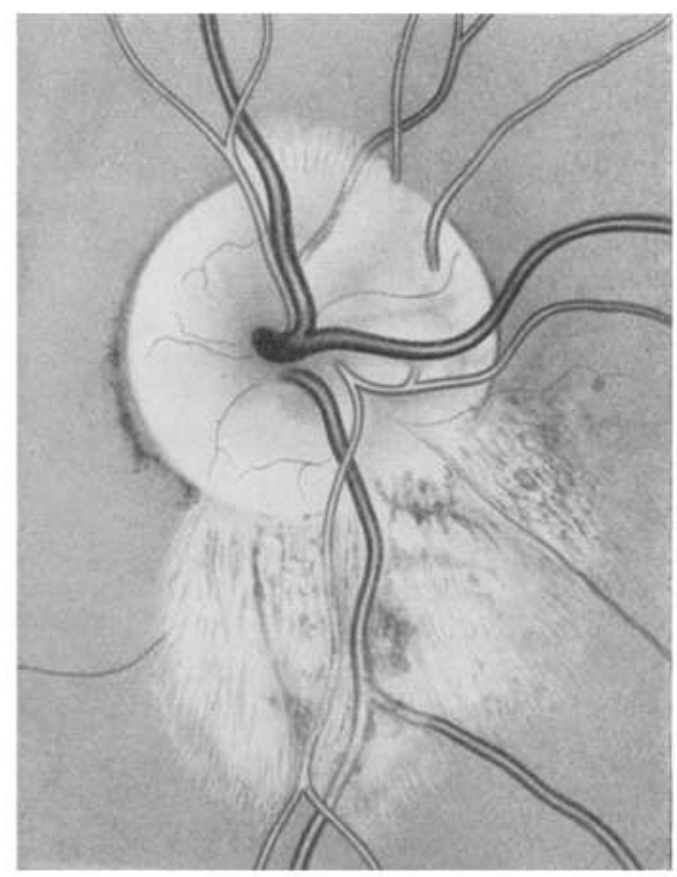

(C)

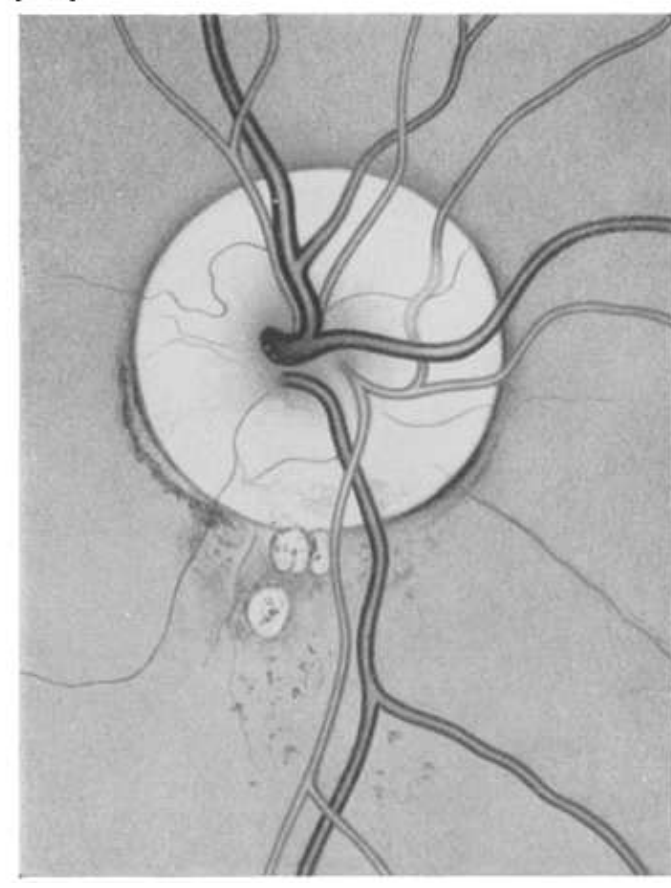

Figure 2: These hand sketched images illustrates probably for the first time the development of axonal degeneration in the retina. At the time, this observation was only apparent thanks to two anatomical oddities coinciding. Firstly the presence of myelinated axons within the retina (a developmental occurrence), seen here as a white bundle on the top and larger white bundle on the bottom of the optic disc. Secondly, occurrence of a central retinal artery occlusion in the same patient which caused retinal axon degeneration over time. (A) A few dark gaps are visible between the inferior bundle of myelinated axons (sketch taken 2 months after sudden loss of vision in the right eye), indicating the beginning of loss of axons and their myelination (B) axonal loss becomes more marked after 4 months and (C) complete optic atrophy is the end result one year after a presumed embolus of the central retinal artery in the 28 year old boy with a mitral valve insufficiency. Bachmann already speculated in 1921 that the mechanism leading to this fundus appearance may be due to ascending (Wallerian) axonal degeneration. Note that the very occasion presence of myelinated retinal axons as seen in this sketch will influence RNFL data acquired by OCT, since intraretinal myelination results in a thicker, more highly reflective RNFL in those areas. MS patients in whom myelinated retinal axons are observed can appear as normal or above normal outliers in treatment trials using OCT as an outcome measure, especially if the myelinated retinal axons are undamaged initially. Reprinted with permission from. ${ }^{40}$ 


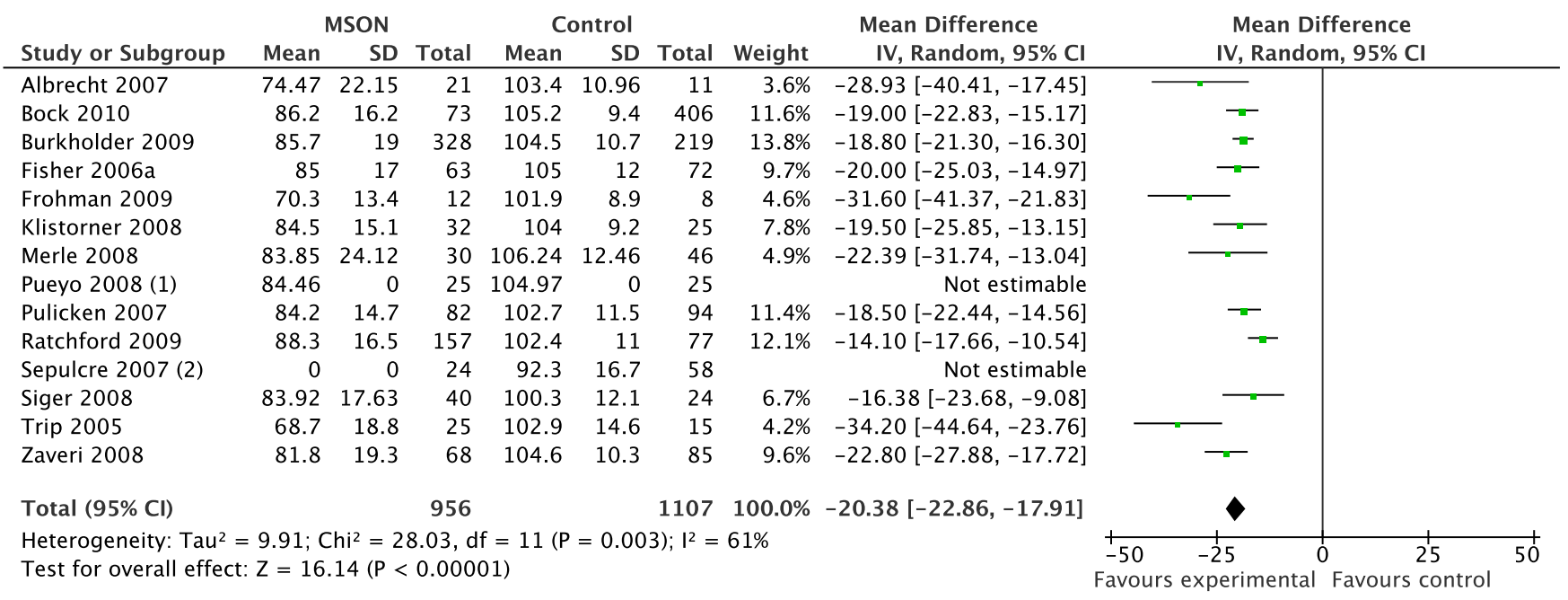

(1) Standard deviation not available from author

(2) Data (mean+/-SD) of ON eyes not published and not available from author.

Figure 3: Meta-analysis of OCT studies in MS patients who did suffer from MSON. The overall averaged RNFL (mean $\pm S D$ ) and number of eyes (not subjects!) investigated is shown for patients and normal subjects. To the right is the data expressed as micron difference in RNFL thickness of eyes that had optic neuritis compared to normal eyes; length of horizontal bar is the $95 \%$ confidence interval for each study. 


\begin{tabular}{|c|c|c|c|c|c|c|c|c|c|c|c|}
\hline \multirow[b]{2}{*}{ Study or Subgroup } & \multicolumn{3}{|c|}{ MS-non-ON } & \multicolumn{3}{|c|}{ Control } & \multirow[b]{2}{*}{ Weight } & \multirow{2}{*}{$\begin{array}{l}\text { Mean Difference } \\
\text { IV, Random, 95\% CI }\end{array}$} & \multirow{2}{*}{\multicolumn{3}{|c|}{$\begin{array}{c}\text { Mean Difference } \\
\text { IV, Random, } 95 \% \mathrm{CI}\end{array}$}} \\
\hline & Mean & SD & Total & Mean & SD & Total & & & & & \\
\hline Albrecht 2007 & 84.59 & 16.03 & 27 & 103.4 & 10.96 & 11 & $2.6 \%$ & $-18.81[-27.67,-9.95]$ & & & \\
\hline Bock 2010 & 97 & 13.1 & 189 & 105.2 & 9.4 & 406 & $12.1 \%$ & $-8.20[-10.28,-6.12]$ & -- & & \\
\hline Burkholder 2009 & 95.6 & 14.5 & 730 & 104.5 & 10.7 & 219 & $12.9 \%$ & $-8.90[-10.66,-7.14]$ & - & & \\
\hline Fisher 2006a & 96 & 14 & 108 & 105 & 12 & 72 & $8.0 \%$ & $-9.00[-12.83,-5.17]$ & & & \\
\hline Frohman 2009 & 101 & 6 & 12 & 101.9 & 8.9 & 8 & $3.7 \%$ & $-0.90[-7.94,6.14]$ & & & \\
\hline Gundogan 2007 & 107.6 & 16.3 & 78 & 110.9 & 10.3 & 76 & $7.1 \%$ & $-3.30[-7.60,1.00]$ & & & \\
\hline Henderson $2008 a$ & 91.12 & 12.6 & 50 & 98.8 & 10.5 & 20 & $5.0 \%$ & $-7.68[-13.46,-1.90]$ & & & \\
\hline Jeanjean 2008 & 88.58 & 13.39 & 7 & 102.34 & 7.47 & 15 & $1.9 \%$ & $-13.76[-24.38,-3.14]$ & & & \\
\hline Klistorner 2008 & 103.8 & 10.8 & 32 & 104 & 9.2 & 25 & $5.7 \%$ & $-0.20[-5.40,5.00]$ & & & \\
\hline Pueyo 2008 (1) & 94.2 & 0 & 75 & 104.97 & 0 & 25 & & Not estimable & & & \\
\hline Pueyo 2009 & 97.93 & 9.08 & 40 & 105.37 & 9.48 & 20 & $6.0 \%$ & $-7.44[-12.46,-2.42]$ & & & \\
\hline Pulicken 2007 & 95.9 & 14 & 202 & 102.7 & 11.5 & 94 & $9.8 \%$ & $-6.80[-9.82,-3.78]$ & & & \\
\hline Ratchford 2009 & 97.4 & 13.9 & 338 & 102.4 & 11 & 77 & $10.2 \%$ & $-5.00[-7.87,-2.13]$ & & & \\
\hline Siger 2008 & 94.38 & 15 & 62 & 100.3 & 12.1 & 24 & $4.6 \%$ & $-5.92[-12.03,0.19]$ & & & \\
\hline Trip 2005 & 94.6 & 14.9 & 25 & 102.9 & 14.6 & 15 & $2.3 \%$ & $-8.30[-17.72,1.12]$ & & & \\
\hline Zaveri 2008 & 95.6 & 15 & 87 & 104.6 & 10.3 & 85 & $8.0 \%$ & $-9.00[-12.84,-5.16]$ & & & \\
\hline Total $(95 \% \mathrm{Cl})$ & & & 1987 & & & 1167 & $100.0 \%$ & $-7.08[-8.65,-5.52]$ & & & \\
\hline $\begin{array}{l}\text { Heterogeneity: } \mathrm{Tau}^{2} \\
\text { Test for overall effec }\end{array}$ & $\begin{array}{l}4.13 \\
Z=8\end{array}$ & $i^{2}=2$ & $\begin{array}{l}9.66, d \\
.0000\end{array}$ & $f=14$ & 0.0 & 8); $1^{2}=$ & $53 \%$ & & $\begin{array}{ll}1 & 1 \\
-20 & -10 \\
\text { rs experimental }\end{array}$ & $\begin{array}{ll} & 1 \\
0 & 10 \\
& \text { Favours }\end{array}$ & $\begin{array}{l}20 \\
\text { ntrol }\end{array}$ \\
\hline
\end{tabular}

(1) Standard deviation not available from author.

Figure 4: Meta-analysis of OCT studies in MS patients who did not suffer from ON, by history. The overall averaged RNFL (mean $\pm S D$ ) and number of eyes investigated is shown, similar to Figure 3 Note that the bar graph to the right, which summarises the difference in RNFL in the asymptomatic eyes compared to normal eyes, shows there is loss of RNFL even in eyes supposedly not suffering from previous optic neuritis. 


\begin{tabular}{|c|c|c|c|c|c|c|c|c|c|c|}
\hline \multirow[b]{2}{*}{ Study or Subgroup } & \multicolumn{3}{|c|}{ MSON eye } & \multicolumn{3}{|c|}{ MS non-ON eye } & \multirow[b]{2}{*}{ Weight } & \multirow{2}{*}{$\begin{array}{l}\text { Mean Difference } \\
\text { IV, Random } 95 \% \mathrm{CI}\end{array}$} & \multirow{2}{*}{\multicolumn{2}{|c|}{$\begin{array}{c}\text { Mean Difference } \\
\text { IV, Random, } 95 \% \mathrm{CI}\end{array}$}} \\
\hline & Mean & SD & Total & Mean & SD & Total & & & & \\
\hline Albrecht 2007 & 74.47 & 22.15 & 21 & 84.59 & 16.03 & 27 & $2.2 \%$ & $-10.12[-21.36,1.12]$ & & \\
\hline Bock 2010 & 86.2 & 16.2 & 73 & 97 & 13.1 & 189 & $4.6 \%$ & $-10.80[-14.96,-6.64]$ & - & \\
\hline Burkholder 2009 & 85.7 & 19 & 328 & 95.6 & 14.5 & 730 & $5.2 \%$ & $-9.90[-12.21,-7.59]$ & & \\
\hline Cheng 2007 & 76.12 & 14.92 & 28 & 96.45 & 11.73 & 33 & $3.5 \%$ & $-20.33[-27.15,-13.51]$ & & \\
\hline Costello 2006 & 77.5 & 29.9 & 54 & 99.8 & 32.5 & 54 & $2.0 \%$ & $-22.30[-34.08,-10.52]$ & & \\
\hline Costello 2008 (1) & 89 & 7.2 & 21 & 105.1 & 3.6 & 21 & $4.8 \%$ & $-16.10[-19.54,-12.66]$ & & \\
\hline Costello 2008a (2) & 78.3 & 15.74 & 27 & 104.4 & 11.29 & 27 & $3.3 \%$ & $-26.10[-33.41,-18.79]$ & & \\
\hline Costello 2009 (3) & 82.3 & 19.7 & 33 & 103.7 & 15.5 & 45 & $3.1 \%$ & $-21.40[-29.50,-13.30]$ & & \\
\hline Fisher $2006 a$ & 85 & 17 & 63 & 96 & 14 & 108 & $4.2 \%$ & $-11.00[-15.96,-6.04]$ & & \\
\hline Frohman 2009 & 70.3 & 13.4 & 12 & 101.8 & 6 & 12 & $3.0 \%$ & $-31.50[-39.81,-23.19]$ & $\leftarrow$ & \\
\hline Garcia-Martin 2010 & 83.27 & 9.5 & 20 & 92.86 & 4.01 & 61 & $4.5 \%$ & $-9.59[-13.87,-5.31]$ & & \\
\hline Grazioli 2008 & 81.7 & 19.2 & 29 & 93.6 & 15.3 & 31 & $2.8 \%$ & $-11.90[-20.72,-3.08]$ & & \\
\hline Klistorner 2008 & 104 & 9.2 & 32 & 103.8 & 10.8 & 32 & $4.3 \%$ & $0.20[-4.72,5.12]$ & & \\
\hline Kochkorov 2009 & 89 & 18 & 16 & 95 & 14 & 24 & $2.4 \%$ & $-6.00[-16.45,4.45]$ & & \\
\hline Laron 2010 & 79.1 & 2.5 & 47 & 96.3 & 1.4 & 65 & $5.5 \%$ & $-17.20[-17.99,-16.41]$ & $\pi$ & \\
\hline Merle 2010 & 80.81 & 18.4 & 31 & 96.7 & 15.8 & 29 & $2.9 \%$ & $-15.89[-24.55,-7.23]$ & & \\
\hline Nakamura 2010 & 84.28 & 14.18 & 19 & 109.45 & 12.78 & 9 & $2.4 \%$ & $-25.17[-35.68,-14.66]$ & & \\
\hline Oreja-Guevara 2010 & 76.42 & 16.87 & 18 & 85.52 & 18.62 & 18 & $2.1 \%$ & $-9.10[-20.71,2.51]$ & & \\
\hline Pulicken 2007 & 84.2 & 14.7 & 82 & 93.9 & 13.1 & 42 & $4.2 \%$ & $-9.70[-14.78,-4.62]$ & & \\
\hline Quelli 2010 & 78.01 & 17.43 & 51 & 95.24 & 11.64 & 65 & $4.0 \%$ & $-17.23[-22.79,-11.67]$ & & \\
\hline Ratchford 2009 & 88.3 & 16.5 & 157 & 97.4 & 13.9 & 338 & $5.0 \%$ & $-9.10[-12.08,-6.12]$ & & \\
\hline Sepulcre 2007 (4) & 85.8 & 13.9 & 122 & 92.3 & 16.7 & 58 & $4.2 \%$ & $-6.50[-11.46,-1.54]$ & & \\
\hline Siepman 2010 & 71.15 & 13.46 & 27 & 90.39 & 13.46 & 38 & $3.6 \%$ & $-19.24[-25.88,-12.60]$ & & \\
\hline Siger 2008 & 83.92 & 17.63 & 20 & 91.08 & 19.3 & 20 & $2.1 \%$ & $-7.16[-18.62,4.30]$ & & \\
\hline Spain 2009 & 75.81 & 5.85 & 24 & 90.93 & 2.95 & 24 & $5.1 \%$ & $-15.12[-17.74,-12.50]$ & & \\
\hline Talman 2010 & 83 & 18 & 208 & 96 & 13 & 381 & $5.0 \%$ & $-13.00[-15.77,-10.23]$ & - & \\
\hline Zaveri 2008 & 81.8 & 19.3 & 68 & 95.6 & 15 & 87 & $4.0 \%$ & $-13.80[-19.37,-8.23]$ & & \\
\hline Total $(95 \% \mathrm{Cl})$ & & & 1631 & & & 2568 & $100.0 \%$ & $-13.84[-15.97,-11.72]$ & & \\
\hline $\begin{array}{l}\text { Heterogeneity: } \mathrm{Tau}^{2}= \\
\text { Test for overall effect }\end{array}$ & $\begin{array}{l}21.31 ; C \\
Z=12.7\end{array}$ & $\begin{array}{l}\text { Chi' } \\
76(P<\end{array}$ & $\begin{array}{l}173.33 \\
0.0000\end{array}$ & $\mathrm{df}=26($ & $(P<0.0$ & $0001)$ & $\mathrm{I}^{2}=85 \%$ & & $\begin{array}{cc}1 & 1 \\
-20 & -10 \\
\text { Favours experimental }\end{array}$ & \begin{tabular}{|ccc} 
& 1 & 1 \\
0 & 10 & 20 \\
l Favours control
\end{tabular} \\
\hline
\end{tabular}

Figure 5: Meta-analysis of OCT studies comparing MSON and nonaffected eyes in MS patients. The overall averaged RNFL (mean $\pm S D$ ) and number of eyes investigated is shown. The difference between the MSON and MS-non-ON eyes shown in this figure is less than the difference between MSON eyes and normal eyes Figure 3 (please note that the scale of the $x$-axis differs between the two Figures). 


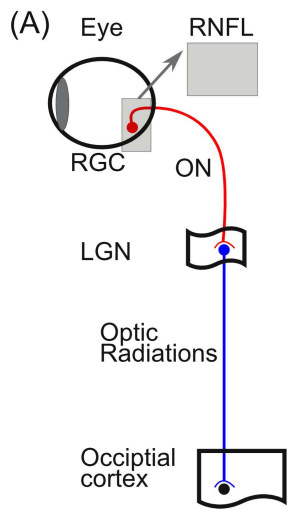

(B)

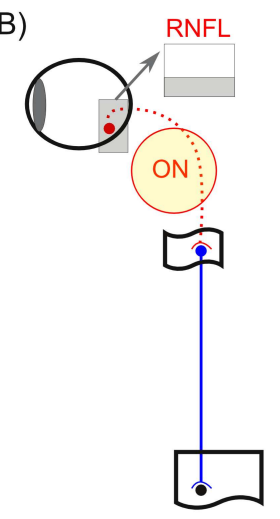

(C)

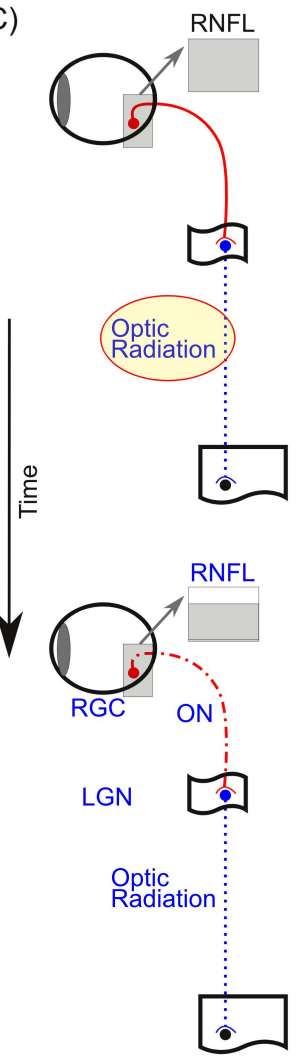

(D)

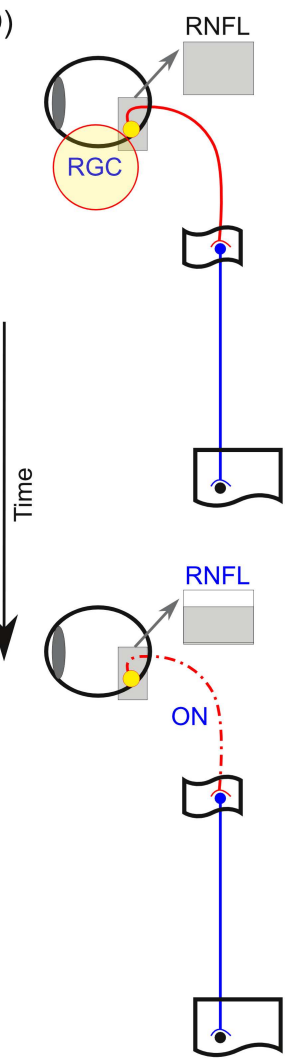

Figure 6: A model of the presumed relationship between RNFL thickness and MS pathology. (A) A simplified sketch of the normal human visual pathways. The retinal ganglion cells (RGC) send their unmyelinated axons into the eye were they form the retinal nerve fibre layer (RNFL, grey inlay), travel to the optic disc and leave the orbit. Once the axons passed the sclera they become myelinated and form the optic nerve (ON). After passing through the chiasma were the temporal fibres cross (not shown) they are called the optic tract. The optic tract wraps around the midbrain and enters the lateral geniculate nucleus (LGN) where all axons must synapse. After the LGN the axons fan out through the deep white matter (optic radiations) to reach the occipital cortex. (B) MSON (red) directly causes acute axonal loss in the ON (red dotted line) leading to marked thinning of the RNFL (small grey box). (C) MS lesions within the optic radiations (blue dotted line) do not immediately result in RNFL thinning. This is thought to be a chBonic consequence of trans-synaptic axonal loss through the LGN. With time (black arrow) trans-synaptic axonal degeneration causes a relative smaller degree of axonal loss in the ON (red dashed-dotted line) with a quantifiable degree of RNFL loss (grey box). (D) Progressive loss of RGC (yellow dot) is a likely result of chronic changes in the anterior visual pathways themselves and causes a small degree of RNFL loss (grey box). 


\section{RNFL thickness over time}

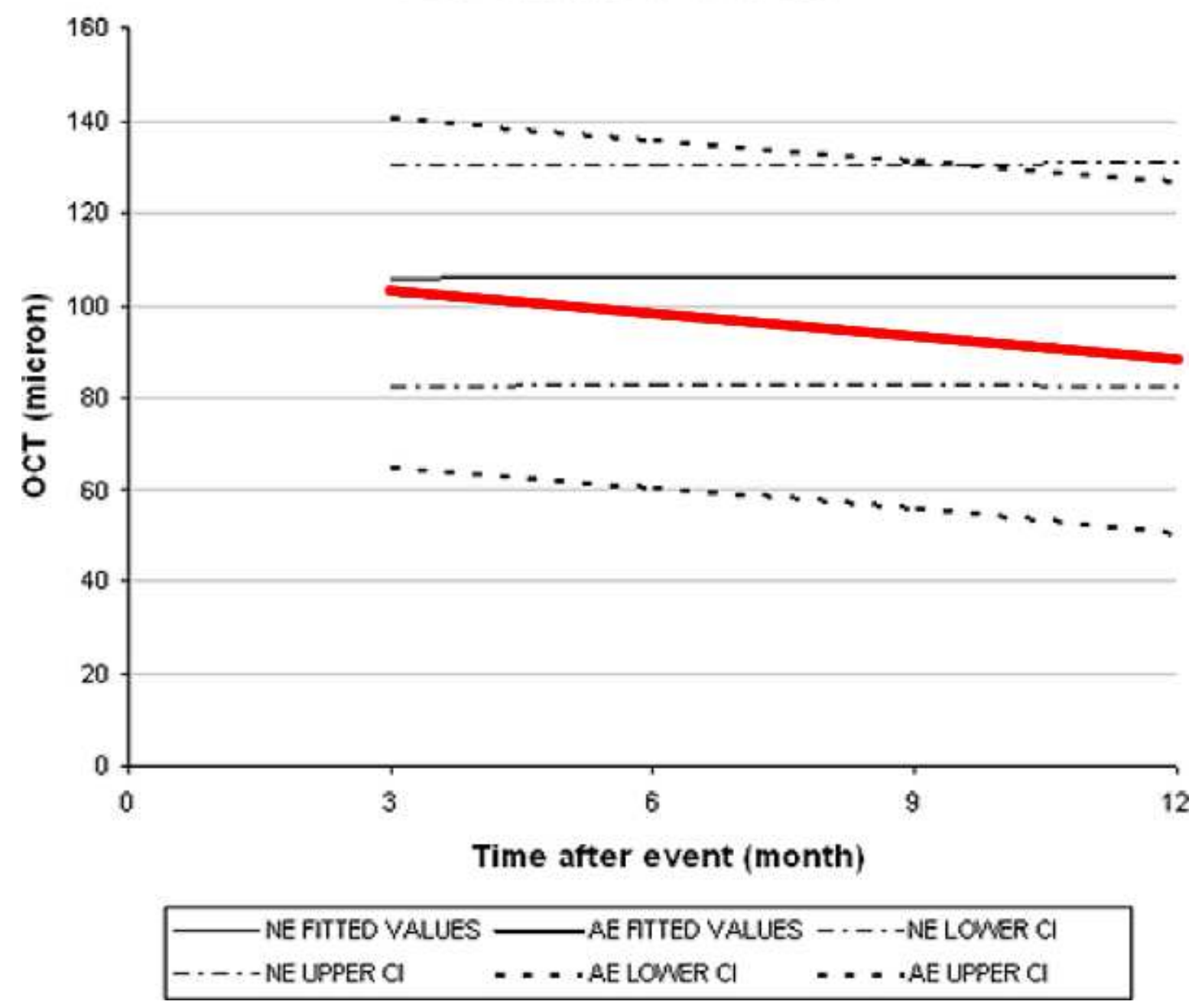

Figure 7: Longitudinal profile of OCT measurements in affected (ON) and non-affected eyes from patients with MS. Image modified from reference ${ }^{7}$ with permission. 